\title{
A Two-Stage Image Segmentation Method for Blurry Images with Poisson or Multiplicative Gamma Noise*
}

\author{
Raymond $\mathrm{Chan}^{\dagger}$, Hongfei Yang $^{\dagger}$, and Tieyong Zeng $\ddagger$
}

\begin{abstract}
In this paper, a two-stage method for segmenting blurry images in the presence of Poisson or multiplicative Gamma noise is proposed. The method is inspired by a previous work on two-stage segmentation and the usage of an I-divergence term to handle the noise. The first stage of our method is to find a smooth solution $u$ to a convex variant of the Mumford-Shah model where the $\ell_{2}$ datafidelity term is replaced by an I-divergence term. A primal-dual algorithm is adopted to efficiently solve the minimization problem. We prove the convergence of the algorithm and the uniqueness of the solution $u$. Once $u$ is obtained, in the second stage, the segmentation is done by thresholding $u$ into different phases. The thresholds can be given by the users or can be obtained automatically by using any clustering method. In our method, we can obtain any $K$-phase segmentation $(K \geq 2)$ by choosing $(K-1)$ thresholds after $u$ is found. Changing $K$ or the thresholds does not require $u$ to be recomputed. Experimental results show that our two-stage method performs better than many standard two-phase or multiphase segmentation methods for very general images, including antimass, tubular, magnetic resonance imaging, and low-light images.
\end{abstract}

Key words. convexity, image segmentation, Gamma noise, multiplicative noise, primal-dual algorithm, total variation

AMS subject classifications. 52A41, 65K10, 65K15, 90C30, 90C47

DOI. $10.1137 / 130920241$

1. Introduction. Image segmentation is an important task in image analysis and computer vision. It aims at separating objects of interest from each other or from the background, or at finding boundaries of such objects. In [50, 51], Mumford and Shah introduced an energy minimization model that allows one to compute an optimal piecewise continuous or piecewise constant approximation $u$ of a given image $f$. Since then, their model has been studied in depth in various aspects, e.g., the properties of minimizers [22] and the approximations and simplifications of their functional and its applications to the problem of image segmentation $[2,1,14,13,16,20,31,49]$.

Denote $\Omega \subset \mathbb{R}^{2}$ as the image domain, and assume that $\Omega$ is bounded, open, and connected, with Lipschitz boundary. Let $f: \Omega \rightarrow \mathbb{R}$ be the given gray scale image to be segmented. In $[50,51]$, Mumford and Shah proposed segmenting $f$ by calculating an optimal approximation

\footnotetext{
${ }^{*}$ Received by the editors May 8, 2013; accepted for publication (in revised form) September 9, 2013; published electronically January 7, 2014.

http://www.siam.org/journals/siims/7-1/92024.html

${ }^{\dagger}$ Department of Mathematics, The Chinese University of Hong Kong, Shatin, NT, Hong Kong (rchan@math.cuhk. edu.hk, yanghongfei@cuhk.edu.hk). The first author's research was supported in part by HKRGC GRF grant CUHK400412, HKRGC CRF grant CUHK2/CRF/11G, CUHK DAG 4053007, and CUHK FIS grant 1902036.

$¥$ Department of Mathematics, Hong Kong Baptist University, Kowloon Tong, Hong Kong (zeng@hkbu.edu.hk). This author's research was supported in part by the National Science Foundation of China (11271049), HKRGC 211710, 211911, and RFGs of HKBU.
} 
$u$ of $f$ and a decomposition of the image domain

$$
\Omega=\Omega_{1} \cup \Omega_{2} \cup \cdots \cup \Omega_{n} \cup \Gamma
$$

such that the following requirements are satisfied: $\Omega_{i}$ 's are disjointly connected open subsets in $\Omega$ with Lipschitz boundaries, $\Gamma$ is the collection of the boundaries of $\Omega_{i}$ 's, and $u$ varies smoothly in $\Omega_{i}$. Then the functional $E$ to be minimized for image segmentation is given by

$$
E(u, \Gamma)=\mathcal{H}^{1}(\Gamma)+\frac{\mu}{2} \int_{\Omega \backslash \Gamma}|\nabla u|^{2} d x+\frac{\lambda}{2} \int_{\Omega}(f-u)^{2} d x,
$$

where $\mathcal{H}^{1}$ denotes the one-dimensional Hausdorff measure in $\mathbb{R}^{2}$; see $[50,51]$.

Because (1.1) is nonconvex, it is very difficult to find or approximate its minimizer. Historically, there are two approaches to studying the minimizer of (1.1). One is to approximate the functional by other functionals. In $[2,1]$, the authors approximated the functional (1.1) by elliptic functionals defined on Sobolev spaces. In $[14,13,16,31,49]$, the authors approximated (1.1) by discrete functionals. Recently, a primal-dual algorithm based on convex relaxation for solving (1.1) was proposed in [53]. This algorithm produces results independent of initializations.

Another approach is to simplify the functional (1.1). For example, if we restrict $\nabla u \equiv 0$ on $\Omega \backslash \Gamma$, then it results in a piecewise constant Mumford-Shah model. In [20], the method of active contours without edges (the Chan-Vese model) for two-phase segmentation was introduced. It solves the piecewise constant Mumford-Shah model but restricts the solution to being a piecewise constant solution with only two constants. For the works on the general piecewise constant Mumford-Shah model, see [38, 62, 63] and the references therein. The main drawback of these methods is that they can easily get stuck in local minima. To overcome the problem, convex relaxation approaches [9, 18, 52], the graph cut method [33], and fuzzy membership functions [41] were proposed.

In [11], the authors proposed a novel two-stage segmentation method that can be considered as a convex variant of the Mumford-Shah model (1.1). In the first stage, a smooth solution $u$ is extracted from the given image $f$ by minimizing the functional

$$
E(u)=\int_{\Omega}|\nabla u| d x+\frac{\mu}{2} \int_{\Omega}|\nabla u|^{2} d x+\frac{\lambda}{2} \int_{\Omega}(\mathcal{A} u-f)^{2} d x .
$$

Here $\mathcal{A}$ is a blurring operator if the given image $f$ is blurred, or it is the identity operator if there is no blur. In the second stage, a thresholding technique is adopted to segment the smooth solution $u$. This model has several advantages. The first is the convexity of the functional (1.2), which guarantees a unique solution that is independent of initializations. The second is that their model can handle multiphase segmentation efficiently. The third advantage is that the thresholding is independent of the process of finding $u$. Users therefore can employ an automated clustering method to find the thresholds, or they can try different phases and thresholds to get a satisfactory segmentation - all without recalculating $u$. One interesting aspect of the model (1.2) is that it closely links three major components of image processing: denoising, deblurring, and segmentation. In fact, model (1.2) has been employed in [35] as an image restoration model. 
Bearing in mind the maximum a posteriori (MAP) approach, the data fitting terms in the Mumford-Shah model in (1.1) and in the model of [11] (i.e., (1.2)) are suitable only for images degraded by additive Gaussian noise. There are in fact many competing methods for segmenting images corrupted by Gaussian noise; see, for instance, [5, 11, 20, 23, 37, 41, $52,57,65,66]$. However, as far as we know, there are only a few works on segmenting images corrupted by Poisson or multiplicative Gamma noise. In [21, 48, 54], the authors proposed snake-based segmentation methods adapted to physical noise of the exponential family (Gaussian, Gamma, Rayleigh, Poisson, etc). In [30], a minimum description length (MDL) criterion was proposed for image segmentation with speckle, Poisson, or Bernoulli noise. In [58], the authors proposed a general segmentation framework for handling additive Gaussian noise, Poisson noise, and multiplicative speckle noise. To the best of our knowledge, there are no papers on the segmentation of blurry images corrupted by Poisson or multiplicative Gamma noise.

Intuitively, for the Mumford-Shah model (1.1) to handle more general noise, one would solve

$$
E(u, \Gamma)=\mathcal{H}^{1}(\Gamma)+\frac{\mu}{2} \int_{\Omega \backslash \Gamma}|\nabla u|^{2} d x+\lambda \mathcal{D}(f, \mathcal{A} u),
$$

where $\mathcal{D}(f, \mathcal{A} u)$ measures the discrepancy between $f$ and $\mathcal{A} u$ according to the noise model. For example, for Poisson noise, $\mathcal{D}(f, \mathcal{A} u)=\int_{\Omega}(\mathcal{A} u-f \log \mathcal{A} u) d x$; see [4], for instance. However, like the original Mumford-Shah model (1.1), it is clear that (1.3) will also be extremely difficult to solve.

Here in this paper, inspired by the work in [11] and [61], we propose a two-stage convex segmentation method to segment blurry images degraded by Poisson or multiplicative Gamma noise. As in [11], in the first stage of our method, we extract a smooth image $u$ from the given image $f$ by minimizing a functional which is a convex variant of (1.3) (just as (1.2) is a convex variant of (1.1)). In the second stage we threshold $u$ to reveal different segmentation features. The functional to minimize in the first stage is

$$
\int_{\Omega}|\nabla u| d x+\frac{\mu}{2} \int_{\Omega}|\nabla u|^{2} d x+\lambda \int_{\Omega}(\mathcal{A} u-f \log \mathcal{A} u) d x .
$$

As observed in [4], the data fitting term $h(u):=\int_{\Omega}(\mathcal{A} u-f \log \mathcal{A} u) d x$ is deduced by MAP probability density $p(\mathcal{A} u \mid f)$ when the image $f$ is blurred by $\mathcal{A}$ and corrupted by Poisson noise (here $p(\cdot \mid \cdot)$ denotes the conditional probability). In [61], the authors used $h(u)$ as the data fitting term and proposed a new model for denoising multiplicative Gamma noise. They gave both theoretical explanations and numerical experiments to justify why $h(u)$ is also suitable for handling multiplicative Gamma noise. Therefore, it is natural for us to introduce the data fitting term $h(u)$ to our two-stage segmentation model when the image is blurry and corrupted by either Poisson or multiplicative Gamma noise.

We will prove that the minimization of the functional (1.4) has a unique solution $u$ which can be solved efficiently by popular algorithms such as the split-Bregman [32] or the Chambolle-Pock algorithm $[17,53]$. When $\mathcal{A}$ is the identity operator, we also show that the unique minimizer of (1.4) will satisfy the maximum principle. One advantage of our method 
is that one does not need to recompute $u$ in the first stage if the threshold in the second stage has to be changed to reveal different features in the image. Another advantage of our approach is that there is no need to specify the number of phases before $u$ is found. We can obtain any $K$-phase segmentation $(K \geq 2)$ by choosing $(K-1)$ thresholds after $u$ is computed in the first stage. In contrast, multiphase methods proposed in $[5,10,26,40,41,42,43,52,59,66]$ require the number of phases to be given first, and if the number of phases changes, one has to solve the minimization problem again.

To the best of our knowledge, our approach is the first for segmenting blurry images corrupted by Poisson or multiplicative Gamma noise, and our numerical results will show that it performs better than many standard two-phase or multiphase segmentation methods for very general images. Furthermore, the numerical scheme we develop here could be regarded as an elegant extension of the standard primal-dual approach in [17], where the underlying problem is a special case of our general setting here (see (4.23) below).

The rest of the paper is organized as follows. In section 2, we briefly review the model in [11] and its properties. In section 3, we introduce our method and show that the minimization model has a unique solution. In section 4 , we give the detailed implementation of our method and show that the resulting algorithm converges. In section 5 , we provide numerical results to verify the effectiveness of our method. In section 6 , we conclude our discussion and point out possible improvements.

2. Review of the model in [11]. Since the model in [11] shares a similar structure with our method, we briefly review it here. The model has two stages. In the first stage, one solves the minimization problem

$$
\inf _{u \in W^{1,2}(\Omega)} E(u)=\left\{\int_{\Omega}|\nabla u| d x+\frac{\mu}{2} \int_{\Omega}|\nabla u|^{2} d x+\frac{\lambda}{2} \int_{\Omega}(f-\mathcal{A} u)^{2} d x\right\},
$$

where $\mu$ and $\lambda$ are positive parameters, $\mathcal{A}$ is a given blurring operator, and $f$ is the given image. After obtaining $u$, which is a smoothed version of $f$, one segments $u$ by a proper thresholding method in the second stage.

This two-stage model is inspired by the Mumford-Shah model and is based on the following observation: one can obtain a good restoration of a binary image by thresholding its smoothed version with a proper threshold; see [11]. Assume that $\Gamma$ is a Jordan curve with measure 0. Let $\Sigma=\overline{\operatorname{Inside}(\Gamma)}$; then $\Gamma=\partial \Sigma$. The objective functional in the Mumford-Shah model (1.1) can be written as

$$
\begin{aligned}
\tilde{E}\left(\Sigma, g_{1}, g_{2}\right)= & \frac{\lambda}{2} \int_{\Sigma \backslash \Gamma}\left(f-g_{1}\right)^{2} d x+\frac{\mu}{2} \int_{\Sigma \backslash \Gamma}\left|\nabla g_{1}\right|^{2} d x+\frac{\lambda}{2} \int_{\Omega \backslash \Sigma}\left(f-g_{2}\right)^{2} d x \\
& +\frac{\mu}{2} \int_{\Omega \backslash \Sigma}\left|\nabla g_{2}\right|^{2} d x+\operatorname{Length}(\Gamma),
\end{aligned}
$$

where $g_{1}$ and $g_{2}$ are defined on $\Sigma \backslash \Gamma$ and $\Omega \backslash \Sigma$, respectively, but are extended smoothly to $\Omega$. Inspired by the proof of Theorem 2 in [18] (see also [9, Theorem 4]), the authors in [11] then proved that a global minimizer of $(2.2)$ with fixed $g_{1}$ and $g_{2}$ can be found by carrying out the minimization

$$
\min _{0 \leq u \leq 1}\left\{\int_{\Omega}|\nabla u| d x+\frac{1}{2} \int_{\Omega}\left\{\lambda\left(f-g_{1}\right)^{2}+\mu\left|\nabla g_{1}\right|^{2}-\lambda\left(f-g_{2}\right)^{2}-\mu\left|\nabla g_{2}\right|^{2}\right\} u(x) d x\right\}
$$


and setting $\Sigma=\{x: u(x) \geq \rho\}$ for $\rho \in(0,1]$ a.e. In this way, the authors in [11] replaced the Length $(\Gamma)$ term by a convex integral term $\int_{\Omega}|\nabla u| d x$ and proposed the first stage as (2.1). Indeed, for some images, one can show theoretically that (2.1) can yield the same solutions as the Mumford-Shah model; see Appendix A.

After $u$ is obtained, the authors in [11] proposed several ways to determine proper thresh$\operatorname{old}(\mathrm{s}) \rho$. For two-phase segmentations, one can set $\rho$ to be the mean of $u$, or a user can try different values of $\rho$ to get the best result. For multiphase segmentation, one can use clustering techniques to determine values of $\rho$ automatically. One such technique is the K-means method [34, 36, 45], and the authors in [11] used it in their numerical experiments for both two-phase and multiphase segmentation. In order to segment the solution $u$ in (2.1) into $K \geq 2$ phases, they first linearly stretched it to $\hat{u}$ such that the intensities of $\hat{u}$ lie in $[0,1]$. Denote $\hat{\rho}_{1} \leq \hat{\rho}_{2} \leq \cdots \leq \hat{\rho}_{K}$ to be the centers of the $K$ clusters of the pixel intensities of $\hat{u}$ obtained by the K-means clustering method. Then one defines the $K-1$ thresholds to be

$$
\rho_{i}=\frac{\hat{\rho}_{i}+\hat{\rho}_{i+1}}{2}, \quad i=1,2, \ldots, K-1 .
$$

Denote $\rho_{0}=0$ and $\rho_{K}=1$; then the $i$ th phase of $\hat{u}, 1 \leq i \leq K$, is given by $\left\{x: \rho_{i-1} \leq \hat{u}(x)<\right.$ $\left.\rho_{i}\right\}$. If the K-means method does not produce a satisfactory segmentation, users can choose the thresholds $\rho$ manually to achieve the best result. Note that changing $\rho$ does not require $u$ to be recomputed.

We remark that another two-stage segmentation method of thresholding smoothed images is proposed in [28]. However, there are major differences between the methods in [11] and [28]. First, the objective functional in the first stage of [11] is convex, while that in the first stage of [28] is not. Second, in the second stage, the threshold used in [11] is determined after the solution $u$ is calculated in the first stage, while the threshold in [28] is calculated in the first stage. As a result, for the method in [11], users can alter the thresholds easily without recalculating the first stage, while for [28], users have to recalculate the first stage if they want to change the thresholds.

3. A two-stage segmentation method for Poisson or multiplicative Gamma noise. Let us first introduce the Poisson noise and the multiplicative Gamma noise. For the Poisson noise, for each pixel $x \in \Omega$ we assume that the intensity $f(x)$ is a random variable following the Poisson distribution with mean $u(x)$; i.e., its probability mass function is

$$
p_{f(x)}(n ; u(x))=\frac{(u(x))^{n} e^{-u(x)}}{n !},
$$

where $n$ is the intensity of $f$ at the pixel $x$. In this case, we say that $f$ is corrupted by Poisson noise.

For the Gamma noise, suppose that for each pixel $x \in \Omega$ the random variable $\eta(x)$ follows the Gamma distribution; i.e., its probability density function is

$$
p_{\eta(x)}(y ; \theta, K)=\frac{1}{\theta^{K} \Gamma(K)} y^{K-1} e^{-\frac{y}{\theta}} \quad \text { for } y \geq 0,
$$

where $\Gamma$ is the usual Gamma function, and $\theta$ and $K$ denote the scale and shape parameters in the Gamma distribution, respectively. Notice that the mean of $\eta(x)$ is $K \theta$, and the variance 
of $\eta(x)$ is $K \theta^{2}$. For multiplicative noise, we assume in general that the mean of $\eta(x)$ equals 1 ; see $[4,24]$. Then we have $K \theta=1$, and its variance is $1 / K$. We assume the degraded image is $f(x)=u(x) \cdot \eta(x)$ and say that $f$ is corrupted by multiplicative Gamma noise.

The data-fidelity term in our method is inspired by the following observations. Suppose that $f$ is the given image with noise following a certain statistical distribution, and let $p(u \mid f)$ be the conditional probability of $u$ when we have observed $f$. Then, based on a MAP approach, restoring the image $u$ is equivalent to maximizing the probability $p(u \mid f)$. Assume that the prior distribution of $u$ is given by

$$
p(u) \propto \exp \left(-\beta \int_{\Omega}|\nabla u| d x\right),
$$

where $\beta$ is a parameter. If the noise follows the Poisson distribution, then maximizing $p(u \mid f)$ corresponds to minimizing the functional

$$
\int_{\Omega}(u-f \log u) d x+\beta \int_{\Omega}|\nabla u| d x
$$

see [39]. If the noise is multiplicative following the Gamma distribution, then maximizing $p(u \mid f)$ corresponds to minimizing the functional

$$
\int_{\Omega}\left(\frac{f}{u}+\log u\right) d x+\beta \int_{\Omega}|\nabla u| d x
$$

see [4]. However, it is observed in the numerical examples in $[4,60]$ that for the denoising model (3.3) the noise survives much longer at low image values if we increase the regularization parameter. Therefore, in [60] the authors suggested taking $w=\log u$ and changing the objective functional (3.3) to

$$
\int_{\Omega}\left(f e^{-w}+w\right) d x+\beta \int_{\Omega}|\nabla w| d x .
$$

In [61], the authors employed the objective functional (3.2) to restore images corrupted by multiplicative Gamma noise, and they justified their selection as follows. First, the gradients of the data fitting term in (3.2) and (3.4) are the same if we use again the relation $w=\log u$. Second, both (3.2) and (3.4) have the same minimizer. Numerical results from [61] also suggest that the functional (3.2) is effective in dealing with multiplicative Gamma noise. Therefore, if we want to segment images corrupted by Poisson noise or multiplicative Gamma noise, it is natural to change the data fitting term $\int_{\Omega}(f-\mathcal{A} u)^{2} d x$ in model $(2.1)$ to $\int_{\Omega}(\mathcal{A} u-f \log \mathcal{A} u) d x$. Then we have the following minimization problem:

$$
\inf _{u \in W^{1,2}(\Omega)} E(u)=\inf _{u \in W^{1,2}(\Omega)}\left\{\int_{\Omega}|\nabla u| d x+\frac{\mu}{2} \int_{\Omega}|\nabla u|^{2} d x+\lambda \int_{\Omega}(\mathcal{A} u-f \log \mathcal{A} u) d x\right\} .
$$

After obtaining $u$ from the minimization problem (3.5), we adopt the same approach as in [11] to get a segmentation of $u$ in the second stage. More precisely, we can try different thresholds to get the best segmentation result, or we can use the K-means clustering method 
to get an automatic algorithm. Here we stress again that there is no need to recompute $u$ if we change the number of phases $K$ or the thresholds $\rho$.

In the following, we study the existence and uniqueness of the solution of (3.5). Since most digital images have predefined ranges, it is natural to assume $f \in L^{\infty}(\Omega)$, and we further assume inf $f>0$. We first study the case when the continuous linear operator $\mathcal{A}$ is the identity operator, i.e.,

$$
\inf _{u \in W^{1,2}(\Omega)} E(u)=\inf _{u \in W^{1,2}(\Omega)}\left\{\int_{\Omega}|\nabla u| d x+\frac{\mu}{2} \int_{\Omega}|\nabla u|^{2} d x+\lambda \int_{\Omega}(u-f \log u) d x\right\} .
$$

Theorem 3.1. Let $\Omega$ be a bounded connected open subset of $\mathbb{R}^{2}$ with a Lipschitz boundary. Let $f \in L^{\infty}(\Omega)$ with $\inf f>0$. Then (3.6) has a unique minimizer $u \in W^{1,2}(\Omega)$ satisfying $0<\inf f \leq u \leq \sup f$.

Proof. For any function $g \in W^{1,2}(\Omega)$, define $g^{+}=\max (g, 0)$ and $g^{-}=-\min (g, 0)$. Then we have $g=g^{+}-g^{-}$. It is clear that $\{u-f \log u\}$ takes its minimum at $u=f$, and it tends to positive infinite when $u \rightarrow \infty$, so $E(u)$ is bounded from below. By taking $u \equiv \mathbf{1} \in W^{1,2}(\Omega)$, we see $E(u)<\infty$, so $E(u)$ is proper. Let $\left\{u_{n}\right\}$ be a minimizing sequence. Then there exists an $M>0$ such that $E\left(u_{n}\right) \leq M$ for all $n \in \mathbb{N}$. Therefore we have

$$
M \geq \int_{\Omega}\left(u_{n}-f \log u_{n}\right) d x \geq-\int_{\Omega}\left(u_{n}-f \log u_{n}\right)^{-} d x
$$

for all $n \in \mathbb{N}$. Since $\left(u_{n}-f \log u_{n}\right)^{-}$is uniformly bounded from above by $|f-f \log f|<\infty$, we conclude that $\int_{\Omega}\left(u_{n}-f \log u_{n}\right) d x$ is uniformly bounded from below. From this and the uniform boundedness of $E\left(u_{n}\right)$, it is clear that both $\left\|\nabla u_{n}\right\|_{1}=\int_{\Omega}\left|\nabla u_{n}\right| d x$ and $\left\|\nabla u_{n}\right\|_{2}=$ $\left(\int_{\Omega}\left|\nabla u_{n}\right|^{2} d x\right)^{\frac{1}{2}}$ are uniformly bounded.

Since $f \in L^{\infty}(\Omega), f$ is bounded from above. Thus for all $y \in \Omega$, there exists a $u_{0}>0$ such that $2(x-f(y) \log x) \geq x$ for $x \geq u_{0}$. Therefore we conclude that

$$
\begin{aligned}
\int_{\Omega}\left|u_{n}\right| d x & \leq \int_{\Omega} \max \left\{2\left(u_{n}-f \log u_{n}\right), u_{0}\right\} d x \leq 2 \int_{\Omega}\left(u_{n}-f \log u_{n}\right)^{+} d x+\int_{\Omega} u_{0} d x \\
& \leq 2 \int_{\Omega}\left(u_{n}-f \log u_{n}\right) d x+2 \int_{\Omega}\left(u_{n}-f \log u_{n}\right)^{-} d x+\int_{\Omega} u_{0} d x<\infty
\end{aligned}
$$

for all $n \in \mathbb{N}$. Thus we have proved that $\left\|u_{n}\right\|_{1}$ is uniformly bounded.

From the Poincaré inequality [27], we have

$$
\left\|u_{n}-m_{\Omega}\left(u_{n}\right)\right\|_{2} \leq C\left\|\nabla u_{n}\right\|_{2},
$$

where $m_{\Omega}\left(u_{n}\right)=\frac{1}{|\Omega|} \int_{\Omega} u_{n} d x,|\Omega|$ is the Lebesgue measure of $\Omega$, and $C$ is a constant related to $\Omega$. Thus $\left\|u_{n}-m_{\Omega}\left(u_{n}\right)\right\|_{2}$ is uniformly bounded. Notice that we have already proved that $\left\|u_{n}\right\|_{1}$ is uniformly bounded. From this we conclude that

$$
\begin{aligned}
\left\|u_{n}\right\|_{2} & \leq\left\|u_{n}-m_{\Omega}\left(u_{n}\right)\right\|_{2}+\left\|m_{\Omega}\left(u_{n}\right)\right\|_{2} \\
& \leq\left\|u_{n}-m_{\Omega}\left(u_{n}\right)\right\|_{2}+\left\|u_{n}\right\|_{1}
\end{aligned}
$$

is uniformly bounded. 
Therefore, up to a subsequence, $u_{n}$ converges strongly in $W^{1,2}(\Omega)$ to some $u^{*}$, and $\nabla u_{n}$ converges weakly as a measure to $\nabla u^{*}$. By the lower semicontinuity of $E(u)$, we have $E\left(\liminf _{n \rightarrow \infty} u_{n}\right) \leq \liminf _{n \rightarrow \infty} E\left(u_{n}\right)$, and therefore $u^{*}$ is a solution to (3.6).

Let $\alpha=\inf f$ and $\beta=\sup f$. By Proposition 15 in [22], both $\min \left(u^{*}, \beta\right)$ and $\max \left(u^{*}, \alpha\right)$ are members of $W^{1,2}(\Omega)$, and

$$
\left|\nabla\left(\min \left(u^{*}, \beta\right)\right)\right| \leq\left|\nabla u^{*}\right|, \quad\left|\nabla\left(\max \left(u^{*}, \alpha\right)\right)\right| \leq\left|\nabla u^{*}\right| .
$$

Then following the same arguments as in Theorem 4.1 in [4], we have $\alpha \leq u^{*} \leq \beta$.

The uniqueness of the minimizer follows from the strict convexity of the objective functional in (3.6).

Next we study the case when $\mathcal{A}$ is a blurring operator. We show that our main model (3.5) has a unique solution if $\operatorname{Ker}(\mathcal{A}) \cap \operatorname{Ker}(\nabla)=\{0\}$, where $\operatorname{Ker}(\cdot)$ represents the kernel. This condition says that $\mathcal{A} \mathbf{1} \neq 0$. In real applications, the blurring operator is a convolution with positive kernel, so the condition $\operatorname{Ker}(\mathcal{A}) \cap \operatorname{Ker}(\nabla)=\{0\}$ is satisfied.

Theorem 3.2. Let $\Omega$ be a bounded connected open subset of $\mathbb{R}^{2}$ with a Lipschitz boundary. Let $f \in L^{\infty}(\Omega)$ with $\inf f>0$, and let $\mathcal{A}$ be a continuous linear operator from $W^{1,2}(\Omega)$ to itself. Assume $\operatorname{Ker}(\mathcal{A}) \cap \operatorname{Ker}(\nabla)=\{0\}$; then (3.5) has a unique minimizer $u \in W^{1,2}(\Omega)$.

Proof. Let $\left\{u_{n}\right\}$ be a minimizing sequence. Then, as argued in the proof of Theorem 3.1, it is clear that $\left\|\nabla u_{n}\right\|_{1},\left\|\nabla u_{n}\right\|_{2}$, and $\left\|\mathcal{A} u_{n}\right\|_{1}$ are all uniformly bounded. By the Poincaré inequality, we have

$$
\left\|u_{n}-m_{\Omega}\left(u_{n}\right)\right\|_{1} \leq C_{1}\left\|\nabla u_{n}\right\|_{1}
$$

for some constant $C_{1}$. Thus $\left\|u_{n}-m_{\Omega}\left(u_{n}\right)\right\|_{1}$ is uniformly bounded. We also have

$$
\begin{aligned}
\left|m_{\Omega}\left(u_{n}\right)\right|\|\mathcal{A} \mathbf{1}\|_{1} & =\left\|\mathcal{A}\left(m_{\Omega}\left(u_{n}\right) \mathbf{1}\right)\right\|_{1} \\
& =\left\|\mathcal{A}\left(m_{\Omega}\left(u_{n}\right)-u_{n}\right)+\mathcal{A} u_{n}\right\|_{1} \\
& \leq\left\|\mathcal{A}\left(m_{\Omega}\left(u_{n}\right)-u_{n}\right)\right\|_{1}+\left\|\mathcal{A} u_{n}\right\|_{1} .
\end{aligned}
$$

Since $\left\|u_{n}-m_{\Omega}\left(u_{n}\right)\right\|_{1}$ is uniformly bounded, $\mathcal{A}$ is continuous, and $\mathcal{A} \mathbf{1} \neq 0$, we see that $m_{\Omega}\left(u_{n}\right)$ is uniformly bounded. By the Poincaré inequality again, we see that

$$
\left\|u_{n}-m_{\Omega}\left(u_{n}\right)\right\|_{2} \leq C_{2}\left\|\nabla u_{n}\right\|_{2}
$$

for some constant $C_{2}$. Thus $\left\|u_{n}\right\|_{2} \leq\left\|u_{n}-m_{\Omega}\left(u_{n}\right)\right\|_{2}+\left\|m_{\Omega}\left(u_{n}\right)\right\|_{2}$ is uniformly bounded. Therefore, up to a subsequence, $u_{n}$ converges strongly in $W^{1,2}(\Omega)$ to some $u^{*}$, and $\nabla u_{n}$ converges weakly as a measure to $\nabla u^{*}$. Then from the lower semicontinuity of $E(u)$, we conclude that $u^{*}$ is a minimizer of (3.5).

Notice that $\mathcal{A} u-f \log \mathcal{A} u$ is strictly convex in $\mathcal{A} u$. Thus the uniqueness of the minimizer follows from the same argument as in [11, Theorem 2.4].

4. The primal-dual algorithm for solving (3.5). Because of the convexity of the minimization problem (3.5), many methods can be used to solve it. For example, we have the primal-dual algorithm $[15,17,19,68]$, which can be easily adapted to a number of nonsmooth convex optimization problems and is easy to implement, and the alternating direction method with multipliers (ADMM) [8, 29], which is convergent and well suited to large-scale 
convex problems. Recently, several specific algorithms for solving TV regularized problems have been proposed, for example, (i) the split-Bregman algorithm [32], which is closely connected to the ADMM method and has fast convergence for TV regularized problems, and (ii) the Chambolle-Pock algorithm [17], which solves a general saddle-point problem based on the primal-dual approach and is fast and flexible and has a known convergent rate. In this paper, we employ the Chambolle-Pock algorithm to solve the minimization problem (3.5).

We now derive the discrete version of (3.5). For the sake of simplicity we keep the same notation from the continuous context. Suppose that the original image $f \in \mathbb{R}^{m n \times 1}$ is obtained from a two-dimensional (2D) pixel-array ( size $m \times n$ ) by concatenation in the usual columnwise fashion, and that $f \in[1,255]$ (we set $f=\max (f, 1)$ ). Define the function $G: \mathbb{R}^{m n \times 1} \rightarrow \mathbb{R}$ as

$$
G(v)=\sum_{i}\left(v_{i}-f_{i} \log v_{i}\right), \quad v>0 .
$$

The discrete gradient operator is the map $\nabla: \mathbb{R}^{m n \times 1} \rightarrow \mathbb{R}^{2 m n \times 1}$ defined as

$$
\nabla u=\left(\begin{array}{c}
\nabla_{x} u \\
\nabla_{y} u
\end{array}\right)
$$

with $\nabla_{x}$ and $\nabla_{y}$ corresponding to the discrete derivative operators in the $x$-direction and $y$-direction, respectively. In our numerical experiments, $\nabla_{x}$ and $\nabla_{y}$ are obtained by applying finite-difference approximations to the derivatives with symmetric boundary conditions in the respective coordinate directions. For example, we define

$$
\left(\nabla_{x} u\right)_{i, j}=\left\{\begin{array}{cl}
u_{i+1, j}-u_{i, j}, & i=1,2, \ldots, n-1, \\
0, & i=n .
\end{array}\right.
$$

In addition, $\|\nabla u\|_{1}$ denotes the discrete total variation of $u$, i.e.,

$$
\|\nabla u\|_{1}=\sum_{i} \sqrt{\left(\nabla_{x} u\right)_{i}^{2}+\left(\nabla_{y} u\right)_{i}^{2}}
$$

Then the discrete version of the minimization problem (3.5) is

$$
\min _{u} E(u)=\min _{u}\left\{\|\nabla u\|_{1}+\frac{\mu}{2}\|\nabla u\|_{2}^{2}+\lambda G(A u)\right\},
$$

where $A \in \mathbb{R}^{m n \times m n}$ is the blurring matrix from the discretization of $\mathcal{A}$. In the numerical tests, we impose symmetric boundary conditions on $A$ too.

Next, we introduce new variables $v \in \mathbb{R}^{2 m n \times 1}$ and $w \in \mathbb{R}^{m n \times 1}$ and reformulate the minimization problem (4.2) as the following constrained optimization problem:

$$
\min _{u, v, w}\left\{\|v\|_{1}+\frac{\mu}{2}\|v\|_{2}^{2}+\lambda G(w)\right\} \quad \text { subject to } v=\left(v_{x}, v_{y}\right)^{T}=\nabla u, w=A u .
$$

To employ the Chambolle-Pock algorithm, we consider the following primal-dual optimization problem:

$$
\min _{u, v, w} \max _{p, q}\left\{\|v\|_{1}+\frac{\mu}{2}\|v\|_{2}^{2}+\lambda G(w)+\langle v-\nabla u, p\rangle+\langle w-A u, q\rangle\right\} .
$$


Then the Chambolle-Pock algorithm is defined through the following iterations:

$$
\begin{gathered}
p^{(k+1)}=\arg \max _{p}\left\{\left\langle\bar{v}^{(k)}-\nabla \bar{u}^{(k)}, p\right\rangle-\frac{1}{2 \sigma}\left\|p-p^{(k)}\right\|_{2}^{2}\right\}, \\
q^{(k+1)}=\arg \min _{q}\left\{\left\langle\bar{w}^{(k)}-A \bar{u}^{(k)}, q\right\rangle-\frac{1}{2 \sigma}\left\|q-q^{(k)}\right\|_{2}^{2}\right\}, \\
u^{(k+1)}=\arg \min _{u}\left\{-\left\langle\nabla u, p^{(k+1)}\right\rangle-\left\langle A u, q^{(k+1)}\right\rangle+\frac{1}{2 \tau}\left\|u-u^{(k)}\right\|_{2}^{2}\right\}, \\
v^{(k+1)}=\arg \min _{v}\left\{\|v\|_{1}+\frac{\mu}{2}\|v\|_{2}^{2}+\left\langle v, p^{(k+1)}\right\rangle+\frac{1}{2 \tau}\left\|v-v^{(k)}\right\|_{2}^{2}\right\}, \\
w^{(k+1)}=\arg \min _{w}\left\{\lambda G(w)+\left\langle w, q^{(k+1)}\right\rangle+\frac{1}{2 \tau}\left\|w-w^{(k)}\right\|_{2}^{2}\right\}, \\
\bar{u}^{(k+1)}=2 u^{(k+1)}-u^{(k)}, \\
\bar{v}^{(k+1)}=2 v^{(k+1)}-v^{(k)}, \\
\bar{w}^{(k+1)}=2 w^{(k+1)}-w^{(k)} .
\end{gathered}
$$

Since the objective functions (4.5)-(4.7) are quadratic, the update of $p, q$, and $u$ can be computed efficiently:

$$
\begin{array}{r}
p^{(k+1)}=\sigma\left(\bar{v}^{(k)}-\nabla \bar{u}^{(k)}\right)+p^{(k)}, \\
q^{(k+1)}=\sigma\left(\bar{w}^{(k)}-A \bar{u}^{(k)}\right)+q^{(k)}, \\
u^{(k+1)}=u^{(k)}+\tau\left(A^{T} q^{(k+1)}-\operatorname{div} p^{(k+1)}\right) .
\end{array}
$$

The solution of (4.8) can be easily obtained by applying the soft thresholding operator. Denoting $t^{(k)}=\frac{\tau}{\mu \tau+1}\left(\frac{1}{\tau} v^{(k)}-p^{(k+1)}\right)$, we have

$$
\begin{aligned}
& v_{x}^{(k+1)}=\max \left\{\left|t^{(k)}\right|-\frac{\tau}{\mu \tau+1}, 0\right\} \cdot \frac{t_{x}^{(k)}}{\left|t^{(k)}\right|}, \\
& v_{y}^{(k+1)}=\max \left\{\left|t^{(k)}\right|-\frac{\tau}{\mu \tau+1}, 0\right\} \cdot \frac{t_{y}^{(k)}}{\left|t^{(k)}\right|},
\end{aligned}
$$


where $\left|t^{(k)}\right|=\sqrt{\left(t_{x}^{(k)}\right)^{2}+\left(t_{y}^{(k)}\right)^{2}}$. The optimality condition for (4.9) gives the quadratic equation

$$
w^{2}+\left(\tau\left(\lambda+q^{(k+1)}\right)-w^{(k)}\right) w-\lambda \tau f=0 .
$$

Its solution is given by

$$
w=\frac{w^{(k)}-\tau\left(q^{(k+1)}+\lambda\right)+\left[\left(\tau\left(\lambda+q^{(k+1)}\right)-w^{(k)}\right)^{2}+4 \tau \lambda f\right]^{1 / 2}}{2} .
$$

The following algorithm summarizes the procedures used to solve the optimization problem (4.2).

Algorithm 1. Solving (4.2) by the Chambolle-Pock algorithm.

1. Initialize: $p^{(0)}=0, u^{(0)}=\bar{u}^{(0)}=f, v^{(0)}=\bar{v}^{(0)}=\nabla f, w^{(0)}=\bar{w}^{(0)}=A u^{(0)}$.

2. Do $k=0,1, \ldots$, until $\frac{\left\|u^{(k)}-u^{(k+1)}\right\|}{\left\|u^{(k+1)}\right\|}<\epsilon$.

(a) Compute $p^{(k+1)}$ by (4.13).

(b) Compute $q^{(k+1)}$ by (4.14).

(c) Compute $u^{(k+1)}$ by (4.15).

(d) Compute $v^{(k+1)}$ by (4.16) and (4.17).

(e) Compute $w^{(k+1)}$ by (4.18).

(f) Update $\bar{u}^{(k+1)}, \bar{v}^{(k+1)}$, and $\bar{w}^{(k+1)}$ by (4.10), (4.11), and (4.12).

3. Output: $u$.

We emphasize that if $A$ is the identity operator, there is no need to introduce $w$ and $q$, and the algorithm can be simplified accordingly.

In the following, we discuss the existence of solution to (4.4) and the convergence of Algorithm 1. Define

$$
K=\left(\begin{array}{ccc}
-\nabla & I & 0 \\
-A & 0 & I
\end{array}\right), \quad x=\left(\begin{array}{c}
u \\
v \\
w
\end{array}\right), \quad \bar{x}=\left(\begin{array}{c}
\bar{u} \\
\bar{v} \\
\bar{w}
\end{array}\right), \quad y=\left(\begin{array}{c}
p \\
q
\end{array}\right) .
$$

Then (4.4) is equivalent to

$$
\min _{x} \max _{y}\{H(x)+\langle K x, y\rangle\},
$$

where $H(x)=\|v\|_{1}+\frac{\mu}{2}\|v\|_{2}^{2}+\lambda G(w)$. First we note the following.

Proposition 4.1. The saddle-point set of (4.19) is nonempty.

The proof follows the same arguments as in [44, Proposition 2].

Next we show that Algorithm 1 converges.

Proposition 4.2. Let $\|K\|_{2}$ be the operator 2-norm of $K$, and let $\left(x^{(n)}, \bar{x}^{(n)}, y^{(n)}\right)$ be defined by Algorithm 1. If we choose $\tau$ and $\sigma$ such that $\tau \sigma<1 /\|K\|_{2}^{2}$, then $\left(x^{(n)}, y^{(n)}\right)$ converges to a saddle point $\left(x^{*}, y^{*}\right)$ of (4.19).

The proposition is a special case of Theorem 1 in [17]. We remark that for the limiting point $\left(x^{*}, y^{*}\right)=\left(u^{*}, v^{*}, w^{*}, p^{*}, q^{*}\right)$, the $u^{*}$ will be the unique solution of (4.2) that we seek. To see that, we first observe that (4.19) is the primal-dual formulation of

$$
\min _{x}\{\iota(K x)+H(x)\},
$$


where $\iota$ is the indicator function of the set $\{0\}$; see [17]. Clearly, by the definition of $K$ and $H$, (4.20) is exactly the same as (4.3). Since (4.2) has a unique solution (under the assumption that $\operatorname{Ker}(A) \cap \operatorname{Ker}(\nabla)=\{0\})$ and $v:=\nabla u$ and $w:=A u$ (see (4.3)), we see that (4.20) has a unique solution too. By [25, Proposition 3.1], if $\left(x^{*}, y^{*}\right)$ is a saddle point of (4.19), then $x^{*}$ is a solution of (4.20), and $x^{*}$ is therefore unique.

Finally we give an estimate of $\|K\|_{2}$.

Proposition 4.3. Denote $\alpha=\sqrt{\|\nabla\|_{2}^{2}+\|A\|_{2}^{2}}$. Then $\|K\|_{2}<\sqrt{\alpha^{2}+1}$.

Proof. To get a bound for the operator $K$, we have

$$
\begin{aligned}
\|K x\|_{2} & =\left\|\left(\begin{array}{c}
-\nabla u+v \\
-A u+w
\end{array}\right)\right\|_{2} \\
& \leq\left\|\left(\begin{array}{c}
-\nabla u \\
-A u
\end{array}\right)\right\|_{2}+\left\|\left(\begin{array}{c}
v \\
w
\end{array}\right)\right\|_{2} \\
& =\sqrt{\|\nabla u\|_{2}^{2}+\|A u\|_{2}^{2}}+\left\|\left(\begin{array}{c}
v \\
w
\end{array}\right)\right\|_{2} \\
& \leq \sqrt{\|\nabla\|_{2}^{2}+\|A\|_{2}^{2}}\|u\|_{2}+\left\|\left(\begin{array}{c}
v \\
w
\end{array}\right)\right\|_{2} .
\end{aligned}
$$

When $\|x\|_{2}=1$, that is, $\|u\|_{2}^{2}+\left\|\left(\begin{array}{c}v \\ w\end{array}\right)\right\|_{2}^{2}=1$, we have

$$
\begin{aligned}
\|K x\|_{2} & \leq \alpha\|u\|_{2}+\left\|\left(\begin{array}{c}
v \\
w
\end{array}\right)\right\|_{2} \\
& \leq \sqrt{\alpha^{2}+1} \cdot \sqrt{\|u\|_{2}^{2}+\left\|\left(\begin{array}{c}
v \\
w
\end{array}\right)\right\|_{2}^{2}} \\
& =\sqrt{\alpha^{2}+1}
\end{aligned}
$$

Thus $\|K\|_{2} \leq \sqrt{\alpha^{2}+1}$. However, we claim that the equality cannot be achieved. To see this, (4.22) is an equality if and only if $\|u\|_{2}=\frac{\alpha}{\sqrt{\alpha^{2}+1}}$ and $\left\|\left(\begin{array}{c}v \\ w\end{array}\right)\right\|_{2}=\frac{1}{\sqrt{\alpha^{2}+1}}$. In this case, $\|u\|_{2}<1$, and (4.21) becomes a strict inequality.

Since $\|\nabla\|_{2}^{2} \leq 8$ (see [15]) and $\|A\|_{2} \leq 1$ (see [44]), we conclude that if $\tau \sigma \leq 0.1$, then Algorithm 1 converges.

Let us remark that the above numerical scheme can be applied to the more general problem

$$
\min _{x} \sum_{i=1}^{n} f_{i}\left(K_{i} x\right)
$$

where for any $i, K_{i}$ is a bounded linear operator from $\mathbb{R}^{p}$ to some $\mathbb{R}^{m_{i}}$ and $f_{i}$ is a proper convex function. Indeed, we can rewrite the above problem as the following equivalent minmax problem:

$$
\max _{y_{1}, \ldots, y_{n}} \min _{x, w_{1}, \ldots, w_{n}}\left\{\sum_{i=1}^{n} f_{i}\left(w_{i}\right)+\sum_{i=1}^{n}\left\langle w_{i}-K_{i} x, y_{i}\right\rangle\right\}
$$


or

$$
\max _{Y} \min _{X}\{f(X)+\langle K X, Y\rangle\}
$$

where $X=\left(x, w_{1}, \ldots, w_{n}\right)^{T}, Y=\left(y_{1}, \ldots, y_{n}\right)^{T}, f(X):=\sum_{i=1}^{n} f_{i}\left(w_{i}\right)$, and

$$
K:=\left(\begin{array}{ccccc}
-K_{1} & I & 0 & \cdots & 0 \\
-K_{2} & 0 & I & \cdots & 0 \\
& & \cdots & & \\
-K_{n} & \cdots & 0 & 0 & I
\end{array}\right) .
$$

We can readily prove that $\|K\|_{2} \leq \sqrt{1+\sum_{i=1}^{n}\left\|K_{i}\right\|_{2}^{2}}$. Now, we can apply the Chambolle-Pock algorithm to (4.24).

5. Numerical experiments. In this section, we compare our method with other segmentation methods. To standardize the experiments, all test images have the range $[1,255]$ (we set $f=\max (f, 1))$, and we always set the mean $K \theta$ of the Gamma distribution to 1 ; see (3.1).

As far as we know, there are no papers on segmenting blurry images with either Poisson or multiplicative Gamma noise. The most recent paper [58] considered two-phase segmentation with additive Gaussian noise, Poisson noise, or multiplicative speckle noise, but with no blur. We will compare this method with the Poisson noise data-fidelity term with our method. In addition, to be more comprehensive, we will compare with methods in $[23,41,65,66]$ which are effective segmentation methods proposed after 2010 for Gaussian noise with $\ell_{2}$ data-fidelity terms. For fair comparisons, we apply the Anscombe transformation to the test images before the implementation of the methods $[23,41,65,66]$. Recall that the Anscombe transformation is defined by $f \rightarrow 2 \sqrt{f+\frac{3}{8}}$; see [3]. Anscombe transformation can stabilize variance, and it has been used in the removal of Poisson noise; see [46].

We note that the method in [58] belongs to the region-based variational segmentation framework. It is not convex, and the segmentation results depend on initializations. The method in [65] uses the continuous max-flow algorithm in [65] and a mimetic finite-difference discretization method [67] to solve the 2D continuous min-cut problem. The method in [66] uses the same algorithm and method to solve the $2 \mathrm{D}$ continuous min-cut problem with multiple labels. The method in [23] is based on tight frames, and the method in [41] is based on fuzzy region competitions. Notice that we could not obtain the code from the authors of [58] before our paper was submitted for publication, so we coded the algorithm on our own. For the methods in $[23,41,65,66]$, the codes are from the authors.

In our method, we put $\tau=4$ and $\sigma=0.025$ for a fast and convergent implementation of Algorithm 1; see the discussion after Proposition 4.3. For images with no blur, we terminate the iteration in Algorithm 1 when $\frac{\left\|u^{(k)}-u^{(k+1)}\right\|}{\left\|u^{(k+1)}\right\|}<10^{-3}$ or the maximum iteration number 600 is reached, while for images with blur, we terminate the iteration in Algorithm 1 when $\frac{\left\|u^{(k)}-u^{(k+1)}\right\|}{\left\|u^{(k+1)}\right\|}<10^{-4}$ or the maximum iteration number 600 is reached. After we get $u$ in (3.5), we choose threshold(s) to segment $u$. The thresholds are chosen by two methods. The first is to use the MATLAB K-means function "KMEANS". This provides an automatic segmentation process. The second method is to choose the threshold manually to produce 
good segmentation results. We use $\rho^{K}$ to denote the thresholds obtained from the "KMEANS" command and $\rho^{U}$ for the thresholds chosen by us. Since $u$ is calculated prior to choosing the number of phases and the thresholds, users can try different numbers of phases and thresholds without recomputing $u$.

For all the segmentation methods used in this section, we tuned the parameters in the experiments to achieve the best visual results. The values of the parameters are given in Appendix B. The boundaries of the results are superimposed on the given images for comparison. All numerical experiments were run on a PC with $2.4 \mathrm{GHz}$ CPU, 4GB RAM, and MATLAB 7.13 (R2011b).

5.1. Two-phase segmentation. In this subsection, we compare our method with the twophase segmentation methods proposed in [23, 58,65].

Example 5.1 (Poisson noise). Figure 1(a) is the original image "Boat" from the Berkeley Segmentation Dataset and Benchmark (see [47]). This image is difficult to segment because of the inhomogeneity of light: the brightness of the water varies, with the top corners being darker, and the boat has both dark and light parts. We corrupted it with Poisson noise to make the segmentation more challenging; see Figure 1(b). Figure 1(f) is the solution $u$ of (3.5) using $\lambda=1$ and $\mu=0.05$, and Figure $1(\mathrm{~g})$ is our segmentation result with threshold $\rho^{K}=142.13$. It can be seen that our method successfully segmented the body and the reflection of the boat. Figure 1(c) created from the method in [65] included the water of the top corners as part of the segmented object. Figure 1(d) from the method in [23] failed to segment the body and the reflection of the boat as a whole. Figure 1(e) from the method in [58] produced a segmentation similar to our result.

To show the importance of the smoothing term $\|\nabla u\|_{2}^{2}$ in (3.5) in our model, we give the result of our method with $\lambda=1$ and $\mu=0$ in Figure 1(h). The thresholds used in both Figures $1(\mathrm{~g})$ and $1(\mathrm{~h})$ are from the K-means clustering method. Notice that in Figure 1(h), the bright part of the boat is excluded from the boat in the segmentation, while in Figure 1(g), the boat and the reflection are included as a whole.

Example 5.2 (multiplicative Gamma noise). Figure 2(a) is the original "Antimass" image. We corrupt it by multiplicative Gamma noise with $K=10$ to obtain Figure 2(b). The bright object in the image (the continental U.S.) does not have a clear cut boundary, and there are many tiny holes in the object, which make it challenging to produce a good segmentation result. Figure 2(f) is the solution $u$ from (3.5) using $\lambda=1$ and $\mu=0.5$. Notice that there is no visible noise left in $u$, and the bright object in the original image is smoothed out. Figures $2(\mathrm{~g})$ and $2(\mathrm{~h})$ are our segmentation results with thresholds $\rho^{K}=92.05$ and $\rho^{U}=20$, respectively. By comparing our results with the results from the methods in [65], [23], and [58] in Figures 2(c), 2(d), and 2(e), respectively, we see that our method can segment the noisy image successfully with both $\rho^{K}$ and $\rho^{U}$, and different meaningful details are revealed. The methods in $[58,65]$ produced results similar to our segmentation with threshold $\rho^{K}$; the method in [23] produced an unnatural boundary.

Example 5.3 (blocky blurry image with Poisson noise). Figure 3(a) is the synthetic image "Shape," which has three clearly separated objects. We first blur it by a vertical motion kernel with length 31 and then corrupt it by Poisson noise; see Figure 3(b). The boundaries of the objects are now blurry and vague so that they are hard to detect. Figure $3(\mathrm{f})$ is the 


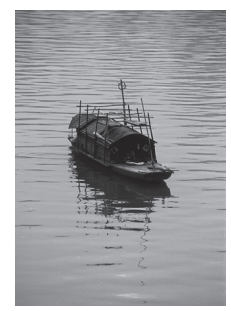

(a) Original image

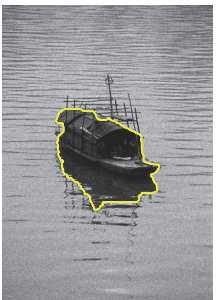

(e) Sawatzky et al. [58]

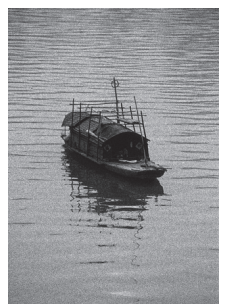

(b) Noisy image

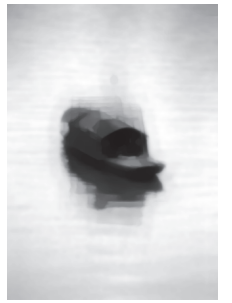

(f) Solution $u$

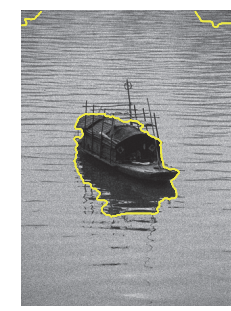

(c) Yuan et al. [65]

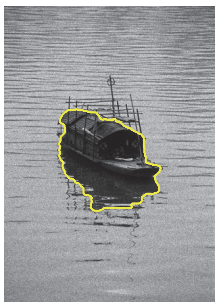

(g) With $\mu=0.05$ and $\rho^{K}=142.13$

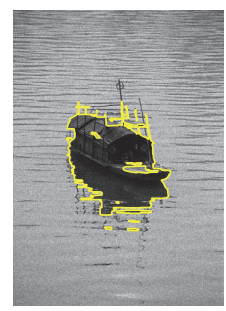

(d) Dong et al. [23]

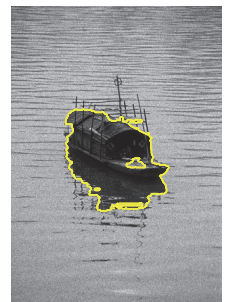

(h) With $\mu=0$ and $\rho^{K}=104.53$

Figure 1. (a) Original "Boat" image $(450 \times 321$ pixels $)$ from the Berkeley Segmentation Dataset and Benchmark (see [47]), (b) image corrupted by Poisson noise, (c) result created from Yuan, Bae, and Tai [65], (d) result from Dong, Chien, and Shen [23], (e) result from Sawatzky et al. [58], (f) solution u from (3.5) with $\lambda=1$ and $\mu=0.05$, (g) $u$ threshold by $\rho^{K}=142.13$, (h) our method with $\lambda=1, \mu=0$, and $\rho^{K}=104.53$.

solution $u$ from (3.5) using $\lambda=15$ and $\mu=0.001$. Figure $3(\mathrm{~g})$ is our segmentation result with threshold $\rho^{K}=129.94$. It is clear that both the blur and the noise are reduced in $u$, and this facilitated the detection of the separate objects. The method in [65] produced the result in Figure 3(c) with oversmoothed boundaries. Because of the blur, Figures 3(d) in [23] and 3(e) in [58] present distortion (see the annulus).

Example 5.4 (tubular blurry image with Gamma noise). Figure 4(a) is the synthetic tubular image "Tree," which resembles a fractal with lots of fine structures. We first blur it by a Gaussian kernel (size $15 \times 15$ and standard deviation 3 ) and then degrade it with multiplicative Gamma noise with $K=10$; see Figure 4(b). Figure 4(f) is the solution $u$ from (3.5) using $\lambda=10$ and $\mu=0.001$. Figures $4(\mathrm{~g})$ and $4(\mathrm{~h})$ are our segmentation results with thresholds $\rho^{K}=48.94$ and $\rho^{U}=14$, respectively. Compared to the methods in [65], [23], and [58] in Figures 4(c), 4(d), and 4(e), respectively, it is clear that our segmentation method with $\rho^{U}=14$ produced a very good result. The methods in $[23,58]$ and our method with $\rho^{K}$ failed to detect fine details of the tree. Because of the blur, the method in [65] produced a very coarse boundary. See Figures 4(i)-4(l) for a detailed comparison.

Since we have the ground truth of Figures 3(a) and 4(a), in Table 1 we compare the percentage of correct pixels of the segmented binary images. Let the image size be $m \times n$, and let the number of correct pixels segmented be $N$. Then the percentage of correct pixels of the segmented image is computed as $\frac{N}{m n}$. We see that our method gives the most accurate segmentation. 


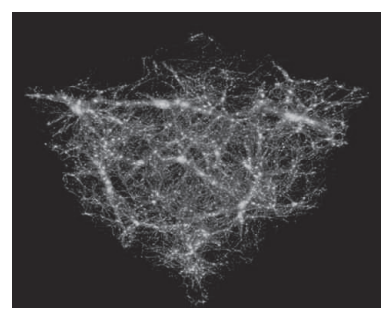

(a) Original image

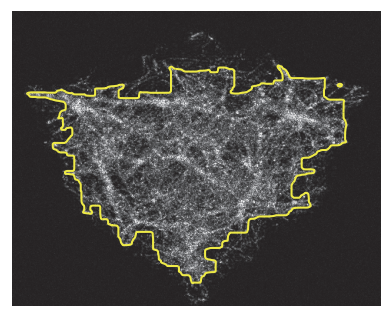

(d) Dong et al. [23]

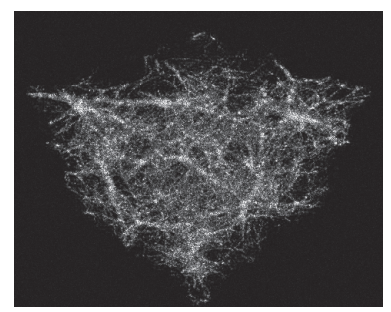

(b) Noisy image

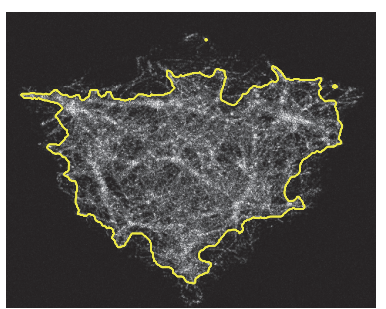

(e) Sawatzky et al. [58]

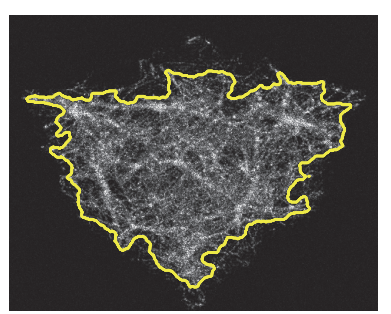

(c) Yuan et al. [65]

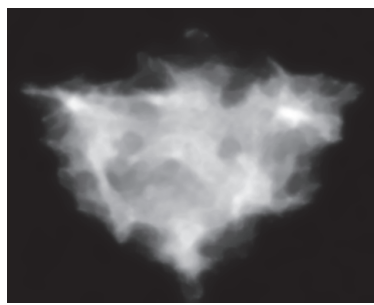

(f) Solution $u$

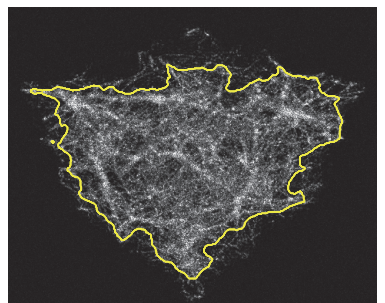

(g) With threshold $\rho^{K}=92.05$

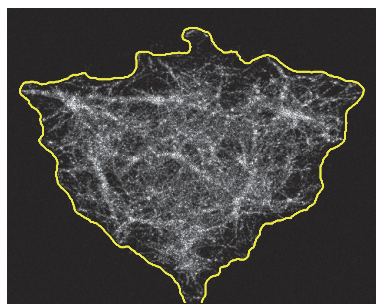

(h) With threshold $\rho^{U}=20$

Figure 2. (a) Original "Antimass" image $(384 \times 480$ pixels), (b) image corrupted by multiplicative Gamma noise with $K=10$, (c) Yuan, Bae, and Tai [65], (d) Dong, Chien, and Shen [23], (e) Sawatzky et al. [58], (f) solution $u$ from (3.5) with $\lambda=1$ and $\mu=0.5$, (g) $u$ with threshold $\rho^{K}=92.05$, (h) $u$ with threshold $\rho^{U}=20$. 


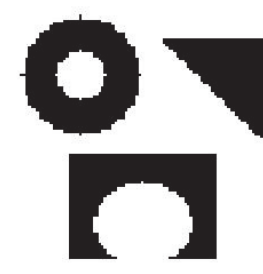

(a) Original image

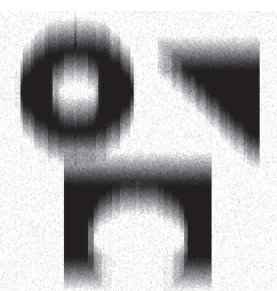

(b) Blurred and noisy image

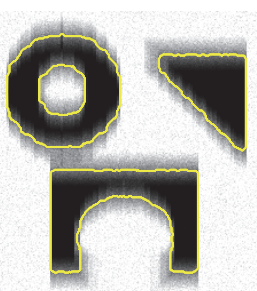

(c) Yuan et al. [65]

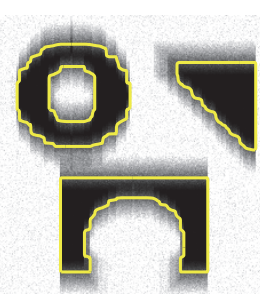

(d) Dong et al. [23]

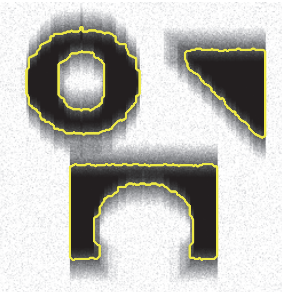

(e) Sawatzky et al. [58]

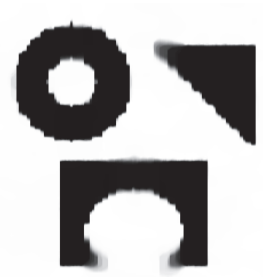

(f) Solution $u$

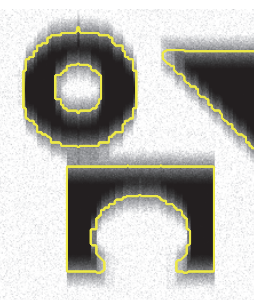

(g) With threshold $\rho^{K}=129.94$

Figure 3. (a) Original "Shape" image $(258 \times 256$ pixels), (b) blurred image (vertical motion kernel with length 31) with Poisson noise, (c) Yuan, Bae, and Tai [65], (d) Dong, Chien, and Shen [23], (e) Sawatzky et al. [58], (f) solution u from (3.5) with $\lambda=15$ and $\mu=0.001$, (g) u threshold with $\rho^{K}=129.94$. 


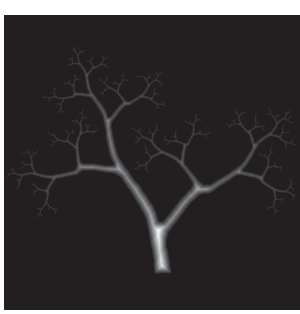

(a) Original image

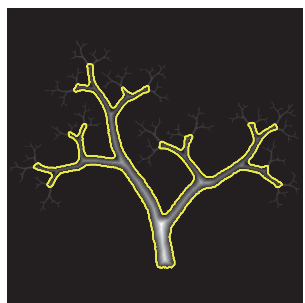

(e) Sawatzky et al. [58]

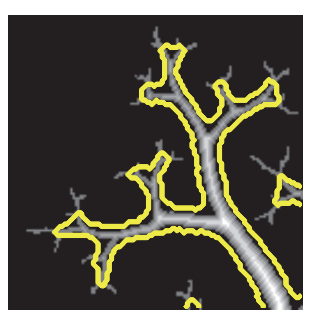

(i) Detail of

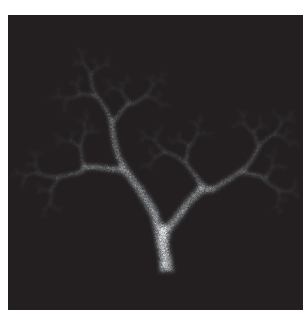

(b) Blurred and noisy image

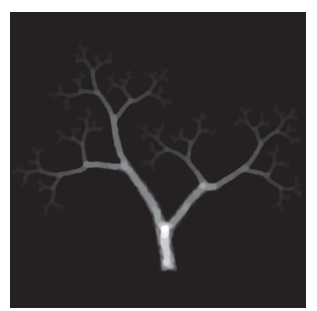

(f) Solution $u$

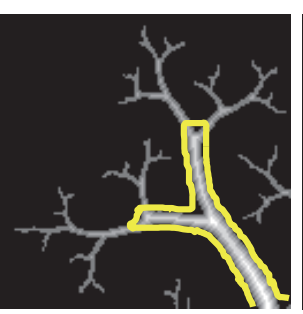

(j) Detail of

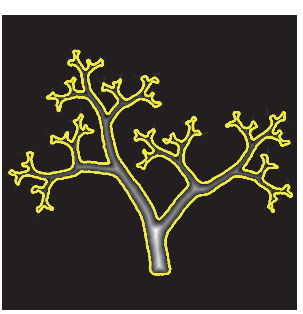

(c) Yuan et al. [65]

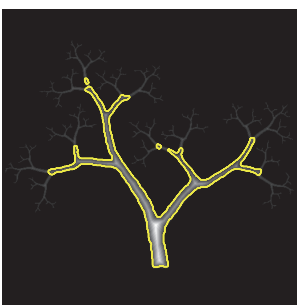

(g) With

threshold $\rho^{K}=48.94$

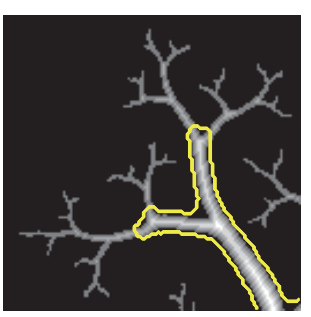

(k) Detail of

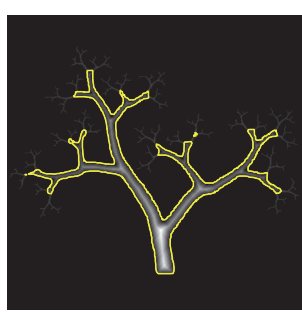

(d) Dong et al. [23]

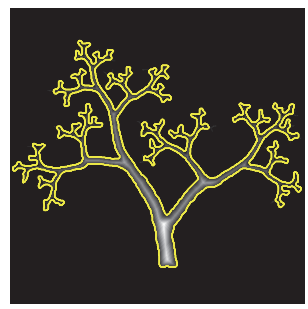

(h) With threshold $\rho^{U}=14$

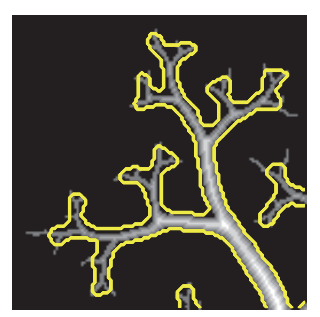

(l) Detail of our method with $\rho^{U}$

Figure 4. (a) Original "Tree" image $(512 \times 512$ pixels), (b) blurred image (Gaussian kernel, standard deviation 3, size $15 \times 15$ ) and multiplicative Gamma noise with $K=10$, (c) Yuan, Bae, and Tai [65], (d) Dong, Chien, and Shen [23], (e) Sawatzky et al. [58], (f) solution $u$ from (3.5) with $\lambda=10$ and $\mu=0.001$, (g) $u$ threshold by $\rho^{K}=48.94$, (h) u threshold by $\rho^{U}=14$, (i) detail of method in [65], (j) detail of method in [23], (k) detail of method in [58], (l) detail of our method with $\rho^{U}$. 
Table 1

Percentage of correct pixels segmented for two-phase segmentation.

\begin{tabular}{|c|c|c|c|c|c|}
\hline & Yuan et al. [65] & Dong et al. [23] & Sawatzky et al. [58] & \multicolumn{2}{|c|}{ Our method } \\
\hline Figure 3 & $97.87 \%$ & $94.47 \%$ & $94.15 \%$ & $\rho^{K}$ & $99.72 \%$ \\
\hline & & & & $\rho^{K}$ & $96.40 \%$ \\
Figure 4 & $95.82 \%$ & $96.90 \%$ & $96.71 \%$ & $\rho^{U}$ & $97.08 \%$ \\
\hline
\end{tabular}

Example 5.5 (real cell image). The noisy image "Cells" in Figure 5(a) is a real image from an automated cell tracking system [6], where the authors developed a system to track cell lineage during Caenorhabditis elegans embryogenesis under low exposure to light. In their experiments, noise in the images led to false positives in nuclear identification. Here, we aim to segment all the cells in the noisy image Figure 5(a). The segmentation result will be useful for further processing, e.g., in locating the cells by circular Hough transform. Figure 5(e) is the $u$ from (3.5) using $\lambda=10$ and $\mu=5$. Figure 5(f) is our segmentation result with threshold $\rho^{K}=55.09$. Figures $5(\mathrm{~g})-5(\mathrm{i})$ are our segmentation results with $\rho^{U}=75,85,95$, respectively, with the same $u$. We stress that by changing the threshold, we do not need to recompute $u$. It is clear that our threshold with $\rho^{K}$ can segment almost all the cells in the noisy image, while our segmentation with $\rho^{U}$ can get more separated cells with increasing thresholds, all with smooth boundaries. For the method in [65], the cells are not separated well. The method in [23] in Figure 5(c) produces a result similar to that of the method of [65] in Figure 5(b). For the method in [58], many cells are left outside the segmented region.

Example 5.6 (real bacteria image). The real "Bacteria" image has intensity only in $[0,48]$; for better visualization we linearly stretched the image to the range [1,255]. The resulting image is depicted in Figure 6(a), where one can see that the object in the image has a high level of noise and the boundary is vague. All the methods we tested are implemented on this linearly stretched image. Figure 6(e) is the solution $u$ from (3.5) using $\lambda=1$ and $\mu=1$. Figures $6(\mathrm{f})$ and $6(\mathrm{~g})$ are our segmentation results with thresholds $\rho^{K}=88.34$ and $\rho^{U}=50$, respectively. By comparing our results with the results from the methods in $[65,23,58]$ in Figures $6(\mathrm{~b})-6(\mathrm{~d})$, we see that our segmentation with $\rho^{U}=50$ produced a boundary that successfully separates the cell from the background. The method in [65] in Figure 6(b) produced a segmentation similar to our result with $\rho^{U}$. The methods in $[23,58]$ did not segment the left end of the object properly.

In Table 2, we give the iteration numbers and CPU time in seconds for our method and the methods in $[23,58,65]$. The codes for all methods, including ours, are written in .mat files. It can be seen that, except for the deblurring cases (Figures 3 and 4), our algorithm uses the least time. The extra time in deblurring cases is justified by the good visual results and higher percentage of correctly segmented pixels; see Table 1.

5.2. Multiphase segmentation. In this section, we compare our method with the multiphase segmentation methods in [66] and [41].

Example 5.7 (multiplicative Gamma noise). Figure 7(a) is the original "Aircraft" image, and we corrupt it by multiplicative Gamma noise with $K=10$ to get Figure 7(b). Figure $7(\mathrm{e})$ is the solution $u$ from (3.5) using $\lambda=2$ and $\mu=0.001$. It is clear that in our solution $u$, the noise is greatly reduced, and the cloud is smoothed out. Figure $7(\mathrm{f})$ is our segmentation 


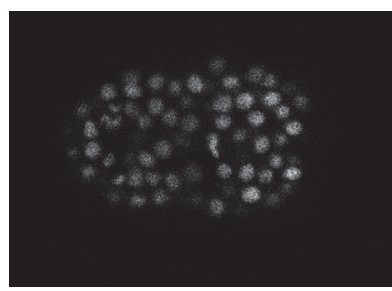

(a) Original noisy image

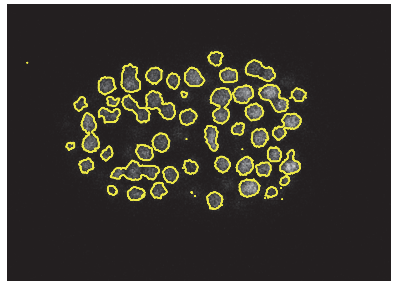

(d) Sawatzky et al. [58

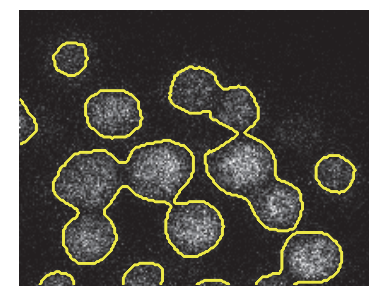

(g) With threshold $\rho^{U}=75$

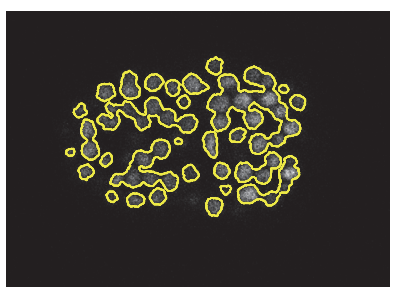

(b) Yuan et al. [65]

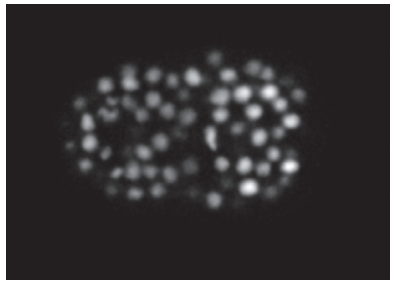

(e) Solution $u$

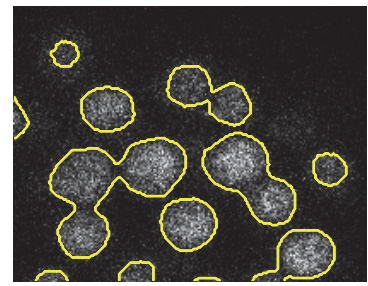

(h) With threshold $\rho^{U}=85$

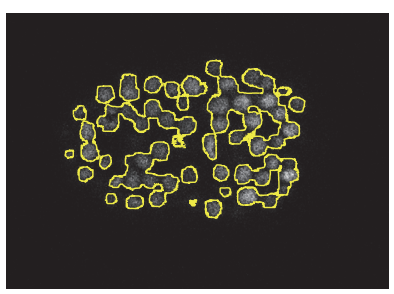

(c) Dong et al. [23]

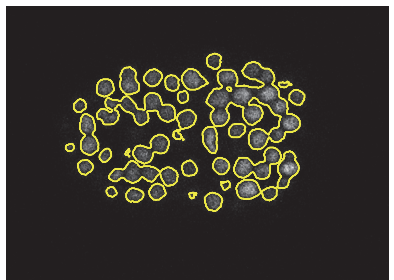

(f) With threshold $\rho^{K}=55.09$

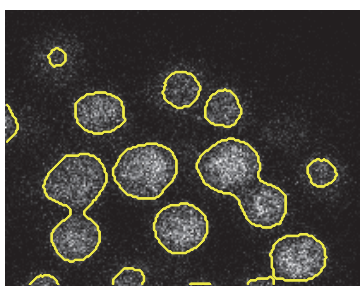

(i) With threshold $\rho^{U}=95$

Figure 5. (a) Original "Cells" image (512 × 712 pixels), (b) Yuan, Bae, and Tai [65], (c) Dong, Chien, and Shen [23], (d) Sawatzky et al. [58], (e) solution $u$ from (3.5) with $\lambda=10$ and $\mu=5$, (f) $u$ threshold with $\rho^{K}=55.09$, (g)-(i) $u$ threshold with $\rho^{U}=75,85,95$, respectively.

result with thresholds $\rho^{K}=(32.46,99.34)$, where the numbers annotate the three different phases. We see that the outline of the airplane is clearly segmented. One can argue that a part of the cloud region marked by the circle in the figure is mislabeled. However, one can preserve that part by choosing $\rho^{U}=(30,80)$; see Figure $7(\mathrm{~g})$. Again we emphasize that the user can choose different thresholds to review different features in the image, all without reminimizing the functional in stage one. One just needs to threshold the solution $u$ in Figure 7 (e) by the new thresholds. Figure 7(c) from the method in [66] is a good segmentation, with the three phases well separated. In Figure 7(d) from the method in [41], although we used the Anscombe transformation, noise is still visible in the segmented image.

Example 5.8 (blocky blurry image with Gamma noise). Figure 8(b) is the blurred and noisy image degraded from the original image in Figure 8(a), first by Gaussian kernel with standard deviation 3 and size 11 and then by multiplicative Gamma noise with $K=20$. Figure 8(e) is the solution $u$ from (3.5) using $\lambda=5$ and $\mu=10^{-5}$. It is clear that our solution $u$ is free of noise, and the blurring is significantly reduced. Figure 8(f) is our segmentation result 


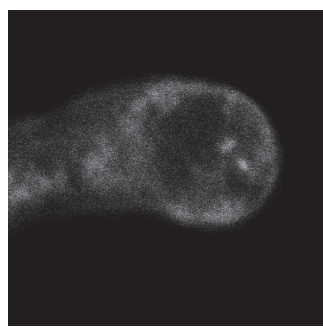

(a) The linearly stretched image

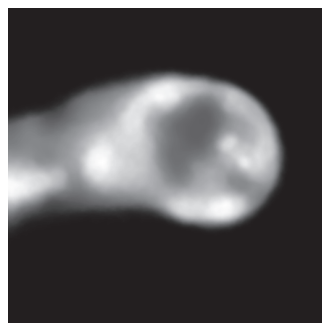

(e) Solution $u$

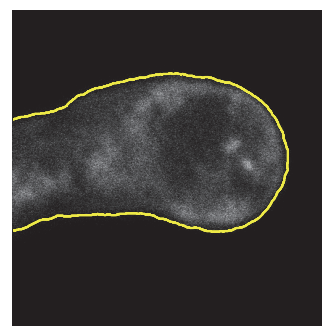

(b) Yuan et al. [65]

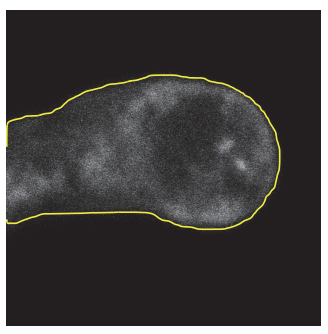

(c) Dong et al. [23]

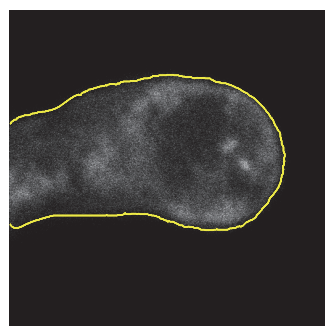

(d) Sawatzky et al.

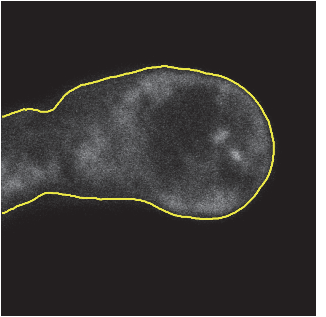

(f) With threshold $\rho^{K}=88.34$

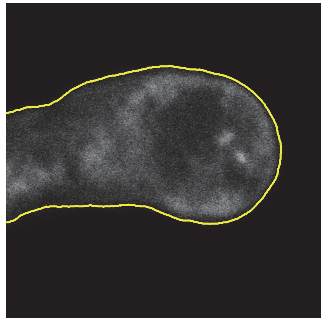

(g) With threshold $\rho^{U}=50$

Figure 6. (a) Original "Bacteria" image after linear stretching (512 $\times 512$ pixels), (b) Yuan, Bae, and Tai [65], (c) Dong, Chien, and Shen [23], (d) Sawatzky et al. [58], (e) solution u from (3.5) with $\lambda=1$ and $\mu=1$, (f) $u$ threshold with $\rho^{K}=88.34$, (g) u threshold with $\rho^{U}=50$.

Table 2

Iteration numbers and CPU time in seconds for two-phase segmentation.

\begin{tabular}{|l|c|c|c|c|r|r|r|r|}
\hline & \multicolumn{2}{|c|}{ Yuan et al. [65] } & \multicolumn{2}{c|}{ Dong et al. [23] } & \multicolumn{2}{|c|}{ Sawatzky et al. [58] } & \multicolumn{2}{|r|}{ Our method } \\
\hline Example & Iter. & Time & Iter. & Time & Iter. & Time & Iter. & Time \\
\hline Figure 1 & 54 & 2.11 & 500 & 189.64 & 13 & 324.52 & 61 & 1.52 \\
\hline Figure 2 & 51 & 5.12 & 500 & 138.01 & 9 & 137.77 & 80 & 3.19 \\
\hline Figure 3 & 22 & 0.17 & 500 & 40.66 & 13 & 37.22 & 325 & 4.12 \\
\hline Figure 4 & 39 & 4.05 & 500 & 190.62 & 14 & 660.13 & 263 & 18.88 \\
\hline Figure 5 & 46 & 6.43 & 500 & 255.50 & 17 & 1546.20 & 101 & 6.26 \\
\hline Figure 6 & 51 & 5.12 & 500 & 189.64 & 12 & 548.69 & 74 & 3.88 \\
\hline
\end{tabular}

with thresholds $\rho^{K}=(42.65,125.87,208.61)$, and Figure $8(\mathrm{~g})$ shows the four different phases. Figure 8(c) from the method in [66] does not segment the brightest part well (see the arrow in the figure), and Figure 8(d) from the method in [41] has visible noise and blurry boundaries.

Example 5.9 (real MRI image). Next we test the four-phase segmentation of a real MRI image; see Figure 9(a). Figure 9(d) is our solution $u$ from (3.5) using $\lambda=10$ and $\mu=0.01$. Figure $9(\mathrm{e})$ is the segmentation of $u$ using the thresholds $\rho^{U}=(110,128,150)$. Figures $9(\mathrm{f})$ 9(i) are the four phases that we segmented. From the images, it is clear that our method produces the best segmentation, while for the methods in [66] and [41], there are holes in the central bright region (marked by a yellow number 4 in Figure $9(\mathrm{e})$ ).

Example 5.10 (image with close varying intensities). Figure 10(a) is the original image, and we corrupt it by Poisson noise to get Figure 10(b). We choose this four-phase image because it 


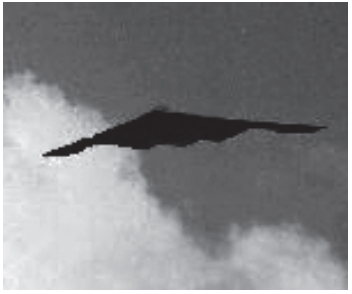

(a) Original image

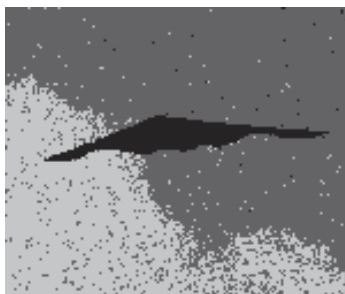

(d) Li et al. [41]

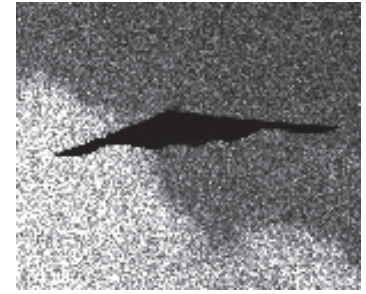

(b) Noisy image

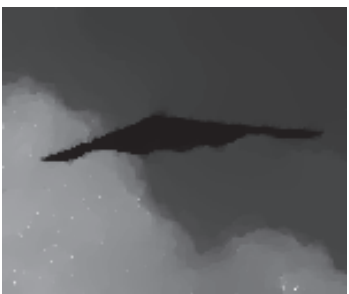

(e) Solution $u$

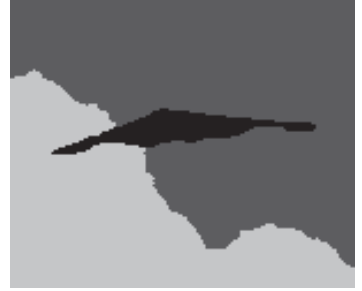

(c) Yuan et al. [66]

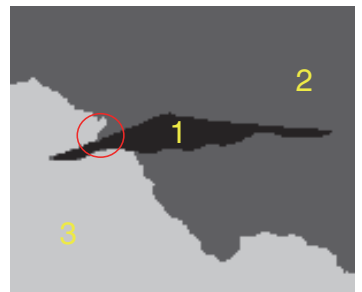

(f) With threshold $\rho^{K}=(32.46,99.34)$

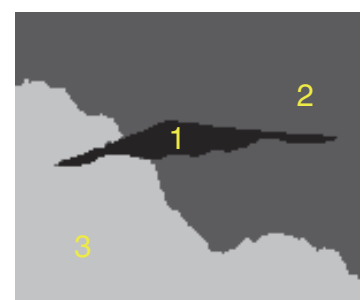

(g) With threshold $\rho^{U}=(30,80)$

Figure 7. (a) Original "Aircraft" image $(125 \times 150$ pixels), (b) image corrupted by multiplicative Gamma noise with $K=10$, (c) Yuan et al. [66], (d) Li et al. [41], (e) solution u from (3.5) with $\lambda=2$ and $\mu=0.001$, (f) $u$ threshold by $\rho^{K}=(32.46,99.34)$, (g) u threshold by $\rho^{U}=(30,80)$.

is difficult to segment. First, the intensities in the four phases are not constant, and phases 1 and 2 have pixels with very close or even the same intensities. Second, unlike in Figure 8, the phases in Figure 10 are not ordered linearly according to their average intensities. In fact, the intensity of phase 2 lies between the intensities of phases 1 and 3, while the intensity of phase 3 lies between the intensities of phases 2 and 4 . Therefore the boundary between phases 1 and 3 , as well as the boundary between phases 2 and 4 , is difficult to detect accurately. To get a good segmentation using our method, we have decreased the stopping tolerance $\epsilon$ for Algorithm 1 to $10^{-5}$. Figure 10(e) is the solution $u$ from (3.5) using $\lambda=1$ and $\mu=10^{-5}$. It is clear that our $u$ is free of noise, with boundaries well preserved and intensities of different phases well separated. Figure 10(f) is our segmentation result with thresholds $\rho^{U}=(73,173,243)$. Because of the close and varying intensities, Figure 10(c) from the method in [66] and Figure 10(d) from [41] fail to separate the two upper regions into two different phases. We remark that we have tried finer convergence criteria for the methods in [66] and [41], but they cannot get better results. 


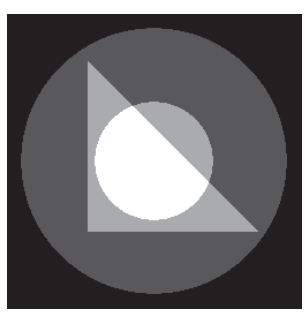

(a) Original image

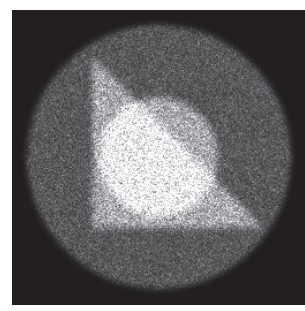

(b) Blurred and noisy image

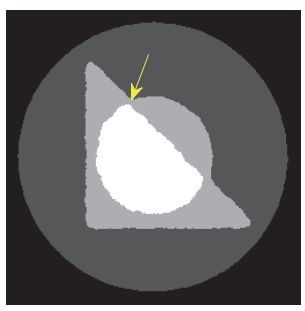

(c) Yuan et al. [66]

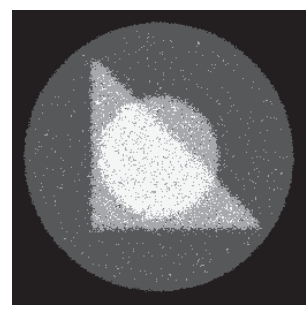

(d) Li et al. [41]

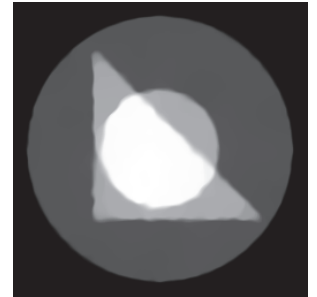

(e) Solution $u$

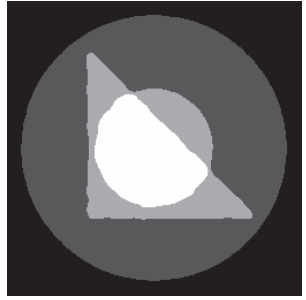

(f) With threshold $\rho^{K}=$ $(42.65,125.87,208.61)$

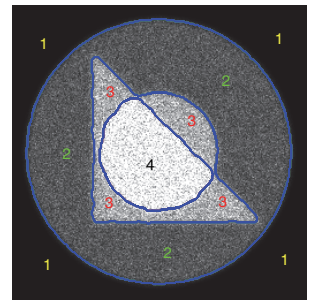

(g) The four phases

Figure 8. (a) Original image, (b) blurred and noisy image: Gaussian kernel with standard deviation 3, size 11, and multiplicative Gamma noise with $K=20$, (c) Yuan et al. [66], (d) Li et al. [41], (e) solution u from (3.5) with $\lambda=5$ and $\mu=10^{-5}$, (f) u threshold by $\rho^{K}=(42.65,125.87,208.61)$, (g) the four phases.

Since we have the ground truth of Figures 8 and 10, in Table 3 we compare the percentage of correct pixels of these two four-phase images. Again, it can be seen that our method produces the best results.

In Table 4, we give the iteration numbers and CPU time in seconds for the multiphase segmentation we tested. The codes for all methods, including ours, are written in .mat files. Except for the deblurring case (Figure 8), our algorithm always uses the least time. Again, the extra time in deblurring is justified by the good visual results and higher percentage of correctly segmented pixels; see Table 3 .

6. Conclusion and possible improvements. In this paper, we have proposed a two-stage method for the segmentation of blurry images with Poisson or multiplicative Gamma noise. In the first stage of our method, we solve the unique smooth minimizer of a convex model (3.5) that is related to the Mumford-Shah model. Then, in the second stage, we segment the image by thresholding the smooth solution of the first stage. Our method has the advantage of solving the two-phase and multiphase segmentations by one single algorithm, and users can decide the number of phases and the thresholds after the solution in (3.5) is obtained. Our numerical experiments show that our method is very effective and robust for many kinds of images, such as antimass, tubular, low-light, noisy, or blurry images.

As pointed out in (1.3), the Mumford-Shah model can be extended to different noise models. However, the resulting functionals will be difficult to minimize. Our approach provides an easy way to construct a convex approximation to these functionals. Judging from the 


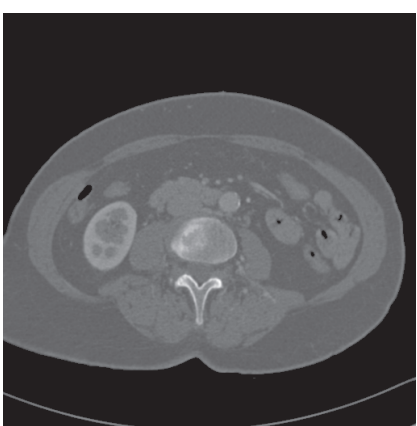

(a) Given image

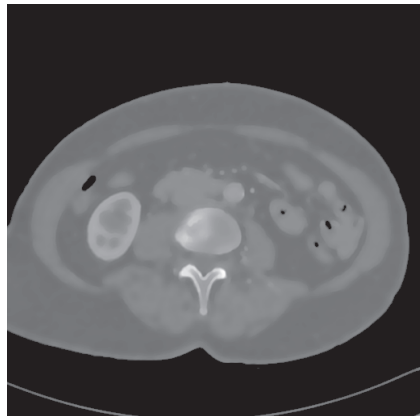

(d) Solution $u$

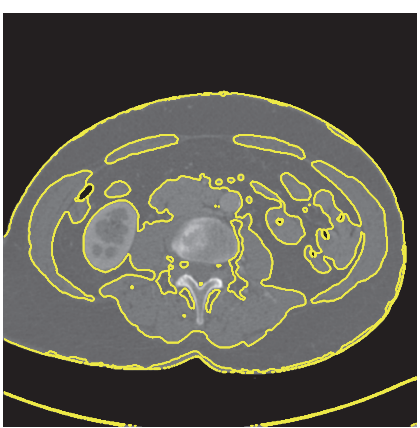

(g) Second phase

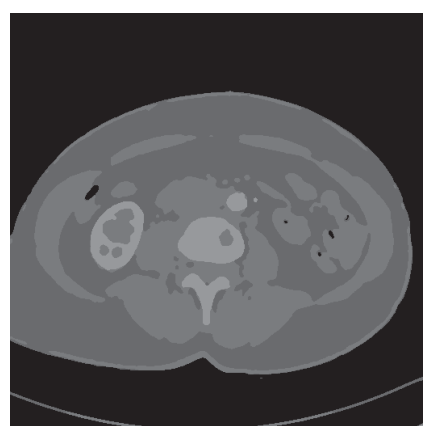

(b) Yuan et al. [66]

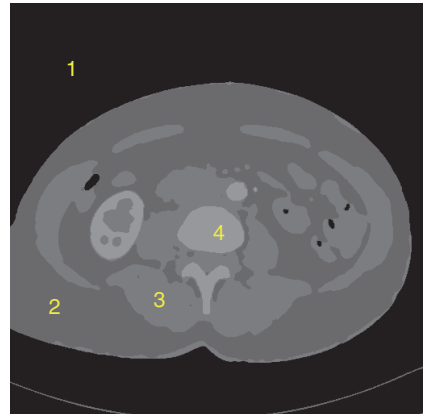

(e) With threshold $\rho^{U}=(110,128,150)$

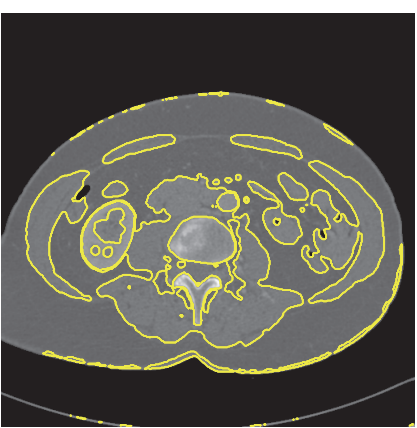

(h) Third phase

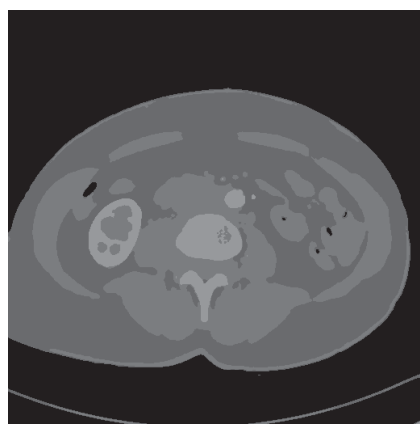

(c) Li et al. [41]

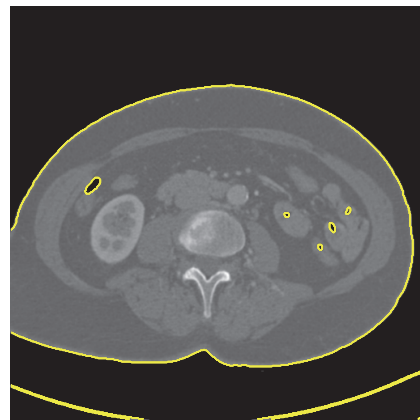

(f) First phase

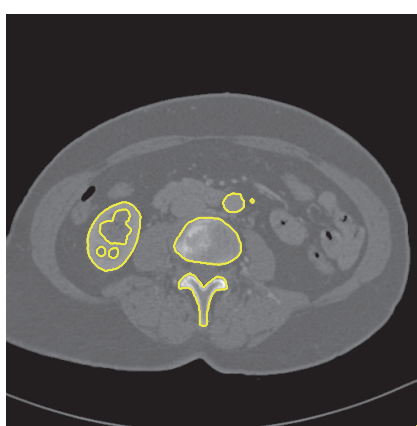

(i) Fourth phase

Figure 9. (a) Original MRI image (512 × 512 pixels), (b) Yuan et al. [66], (c) Li et al. [41], (d) solution $u$ from (3.5) with $\lambda=10$ and $\mu=0.01$, (e) $u$ threshold by $\rho^{U}=(110,128,150)$, (f)-(i) the four phases.

numerical results in this paper and those in [11], we believe that the resulting segmentation algorithm will be an efficient and accurate one.

Our method may be further improved in several ways. One is to employ automatic clustering algorithms other than the K-means method to find the thresholds. Another way of improving the method is to include local information in the clustering process in the second stage to better distinguish different objects of interest. Third, we may try to employ automatic methods, e.g., [64], to determine the parameters $\mu$ and $\lambda$ in the objective functional (3.5). 


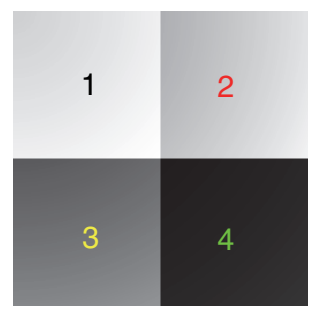

(a) Original image

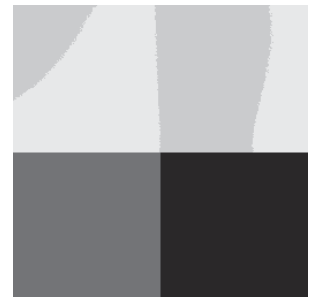

(d) Li et al. [41]

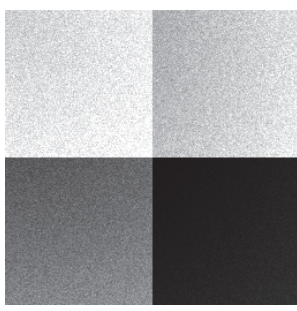

(b) Noisy image

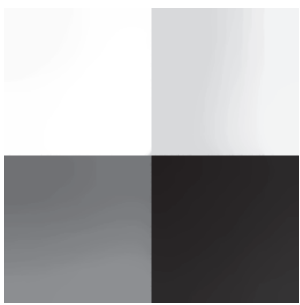

(e) Solution $u$

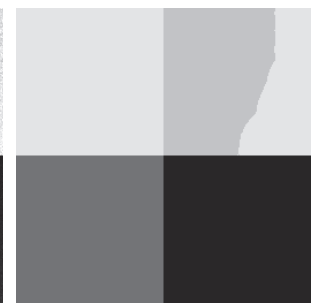

(c) Yuan et al. [66]

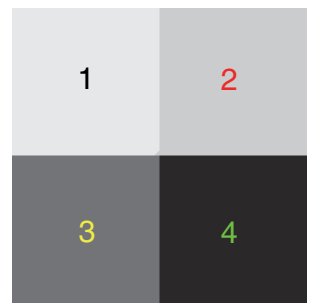

(f) with

threshold $\rho^{U}=$

$(73,173,243)$

Figure 10. (a) Original image (256 $\times 256$ pixels), (b) image corrupted by Poisson noise, (c) Yuan et al. [66], (d) Li et al. [41], (e) solution u from (3.5) with $\lambda=1$ and $\mu=10^{-5}$, (f) u threshold by $\rho^{U}=(73,173,243)$.

Table 3

Percentage of correct pixels segmented in four-phase segmentation.

\begin{tabular}{|l|c|c|c|c|}
\hline & Yuan et al. [66] & Li et al. [41] & \multicolumn{2}{|c|}{ Our method } \\
\hline Figure 8 & $97.00 \%$ & $93.87 \%$ & $\rho^{K}$ & $99.47 \%$ \\
\hline Figure 10 & $91.70 \%$ & $86.60 \%$ & $\rho^{U}$ & $99.99 \%$ \\
\hline
\end{tabular}

Table 4

Iteration numbers and CPU time in seconds for multiphase segmentation.

\begin{tabular}{|l|r|r|r|r|r|r|}
\hline & \multicolumn{2}{|c|}{ Yuan et al. [66] } & \multicolumn{2}{c|}{ Li et al. [41] } & \multicolumn{2}{c|}{ Our method } \\
\hline Example & Iter. & Time & Iter. & Time & Iter. & Time \\
\hline Figure 7 & 127 & 0.97 & 95 & 1.00 & 86 & 0.22 \\
\hline Figure 8 & 57 & 2.19 & 49 & 1.61 & 184 & 2.29 \\
\hline Figure 9 & 76 & 25.67 & 114 & 26.42 & 19 & 0.62 \\
\hline Figure 10 & 114 & 4.37 & 332 & 9.85 & 444 & 2.97 \\
\hline
\end{tabular}

Appendix A. Mumford-Shah model and model in [11]. In this appendix, we show by an example that with suitably chosen parameters, the model in [11] (i.e., (2.1)) can generate the same solutions as the Mumford-Shah model (1.1). For simplicity, we let $A=I$.

We consider the segmentation of the image $f=a \chi_{B(0,1)}$ defined on the domain $\Omega=\mathbb{R}^{2}$, where $a$ is a positive constant and $B(0,1)$ represents the open ball centered at 0 with radius 1. We choose this image because the corresponding Mumford-Shah model (1.1) has explicit solutions. Indeed, according to [22], there are two minimizer pairs $(u, \Gamma)$ of the Mumford-Shah 
model: (i) $\Gamma=\partial B(0,1)$ and $u=a \chi_{B(0,1)}$, and (ii) $\Gamma=\emptyset$ and $u \in W^{1,2}(\Omega)$ is the minimizer of

$$
\min _{u}\left\{\frac{\lambda}{2} \int_{\Omega}\left|u-a \chi_{B(0,1)}\right|^{2}+\frac{\mu}{2} \int_{\Omega}|\nabla u|^{2}\right\}
$$

For the first pair, a segmentation is produced with $\Gamma=\partial B(0,1)$. For the second pair, we have $u=\Psi *\left(a \chi_{B(0,1)}\right)$, where $*$ stands for convolution and the Fourier transform of $\Psi$ is known: $\mathcal{F} \Psi(\xi)=\frac{\lambda}{\lambda+4 \pi^{2} \mu \xi^{2}}$. This $u$ can be regarded as a smooth version of $a \chi_{B(0,1)}$.

We now show that for both minimizer pairs, the two-stage segmentation (2.1) can yield essentially identical results with proper selection of parameters. According to [12], for an input image of the form $f=a \chi_{B(0,1)}$, solving the model (2.1) with $\mu=0$ gives the solution $u=\max \left\{a-\frac{2}{\lambda}, 0\right\} \chi_{B(0,1)}$. Thus if $\lambda>\frac{2}{a}$, this would produce exact segmentation of the disk with boundary $\partial B(0,1)$, just like that of the first minimizer pair. On the other hand, if both $\lambda$ and $\mu$ tend to infinity, then the $\int_{\Omega}|\nabla u| d x$ term will be negligible, and (2.1) will reduce to (A.1), and hence its solution will coincide with that of the second minimizer pair.

In Figure 11, we show numerically that the minimization pairs for the Mumford-Shah (MS) model (1.1) with $\lambda=5$ and $\mu=5$ can be well approximated by solutions of (2.1). Figure 11(a) is the original image, with the intensity of the middle horizontal line plotted in Figure 11(f). Figure 11(b) is the $u$ of the first minimizer pair of the MS model with $\Gamma=\partial B(0,1)$, and Figure 11(c) is the solution of (2.1) with $\lambda=5$ and $\mu=0$. It can be seen that these two images are almost the same, and the intensity plots in (g) and (h) both preserve the boundaries. Figure 11(d) is the $u$ of the second minimizer pair of the MS model with $\Gamma=\emptyset$, and Figure 11(e) is the solution of (2.1) with $\lambda=5$ and $\mu=5$. From the intensity plots in Figures 11(i) and 11(j), it can be seen that these images are very similar and that only points close to the boundaries are smoothed. We stress that both Figures 11(b) and 11(d) correspond to exact minimizer pairs of the MS model.

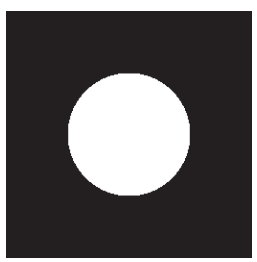

(a) Original image

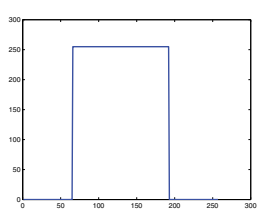

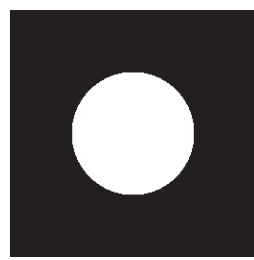

(b) MS model, $\Gamma=\partial B(0,1)$

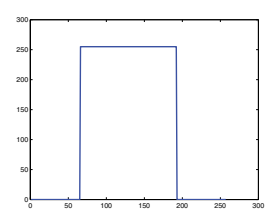

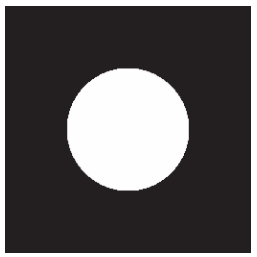

(c) Solution with $\mu=0$

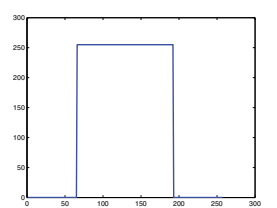

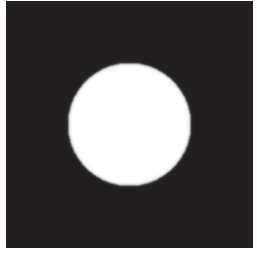

(d) MS model, $\Gamma=\emptyset$

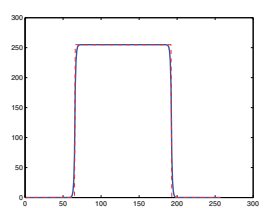

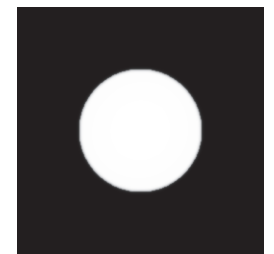

(e) Solution with $\mu=5$

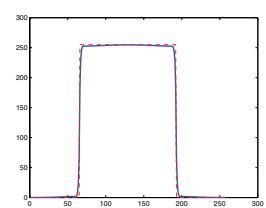

(f) intensity of (a) (g) intensity of (b) (h) intensity of (c) (i) intensity of (d) (j) intensity of (e)

Figure 11. (a) Original image $(257 \times 257$ pixels), (b) u of $M S$ model, $\Gamma=\partial B(0,1)$, (c) $u$ of (2.1) with $\lambda=5, \mu=0$, (d) $u$ of $M S$ model, $\Gamma=\emptyset$, (e) $u$ of (2.1) with $\lambda=5, \mu=5$, (f)-(j) intensity plots for (a)-(e). 
Appendix B. Parameters used in the tests. In Tables 5 and 6 we list the parameters used in the tests for the methods we compared with our method, i.e., those in $[23,41,58,65,66]$. Notice that we could not obtain the code for [58] from the authors before our paper was submitted for publication, so we coded the method on our own. For parameters not listed here, we used the default values in the codes given by the authors.

\section{Table 5}

Parameters of Yuan, Bae, and Tai [65], Dong, Chien, and Shen [23], and Sawatzky et al. [58] for two-phase segmentation.

\begin{tabular}{|l|l|l|l|}
\hline & Yuan et al. [65] & Dong et al. [23] & Sawatzky et al. [58] \\
\hline Figure 1 & ulab $=(0.5,0.8), \alpha=0.5$ & $\mu=20$ & $\alpha_{1}=0.8, \alpha_{2}=0.4, \beta=120$ \\
\hline Figure 2 & ulab $=(0.1,0.4), \alpha=0.5$ & $\mu=35$ & $\alpha_{1}=10, \alpha_{2}=10, \beta=110$ \\
\hline Figure 3 & ulab $=(0.35,0.8), \alpha=0.1$ & $\mu=20$ & $\alpha_{1}=10, \alpha_{2}=50, \beta=50$ \\
\hline Figure 4 & ulab $=(0,0.35), \alpha=0.1$ & $\mu=100$ & $\alpha_{1}=10, \alpha_{2}=50, \beta=40$ \\
\hline Figure 5 & ulab $=(0,0.45), \alpha=0.4$ & $\mu=20$ & $\alpha_{1}=10, \alpha_{2}=50, \beta=50$ \\
\hline Figure 6 & ulab $=(0,0.3), \alpha=1$ & $\mu=5$ & $\alpha_{1}=10, \alpha_{2}=50, \beta=200$ \\
\hline
\end{tabular}

Table 6

Parameters of Yuan et al. [66] and Li et al. [41] for multiphase segmentation.

\begin{tabular}{|l|l|l|}
\hline & Yuan et al. [66] & Li et al. [41] \\
\hline Figure 7 & ulab $=(0,0.3,0.6), \alpha=0.1$ & $\lambda=0.01$ \\
\hline Figure 8 & ulab $=(0,0.37,0.5,0.74), \alpha=0.1$ & $\lambda=0.02$ \\
\hline Figure 9 & ulab $=(0.2,0.56,0.8,0.84), \alpha=0.1$ & $\lambda=0.001$ \\
\hline Figure 10 & ulab $=(0,0.6,0.67,0.74), \alpha=0.05$ & $\lambda=0.015$ \\
\hline
\end{tabular}

\section{REFERENCES}

[1] L. Ambrosio And V. M. Tortorelli, Approximation of functionals depending on jumps by elliptic functionals via $\Gamma$-convergence, Comm. Pure Appl. Math., 43 (1990), pp. 999-1036.

[2] L. Ambrosio and V. M. Tortorelli, On the approximation of free discontinuity problems, Boll. Un. Mat. Ital. B (7), 6 (1992), pp. 105-123.

[3] F. J. Anscombe, The transformation of Poisson, binomial and negative-binomial data, Biometrika, 35 (1948), pp. 246-254.

[4] G. Aubert And J.-F. Aujol, A variational approach to removing multiplicative noise, SIAM J. Appl. Math., 68 (2008), pp. 925-946.

[5] E. BAE, J. YuAn, AND X.-C. TAI, Simultaneous convex optimization of regions and region parameters in image segmentation models, in Innovations for Shape Analysis, Math. Vis., Springer-Verlag, Berlin, Heidelberg, 2013, pp. 421-438.

[6] Z. Bao, J. I. Murray, T. Boyle, S. L. Ooi, M. J. Sandel, and R. H. Waterston, Automated cell lineage tracing in Caenorhabditis elegans, Proc. Natl. Acad. Sci. USA, 103 (2006), pp. 2707-2712.

[7] L. Bar, T. Chan, G. Chung, M. Jung, N. Kiryati, R. Mohieddine, N. Sochen, and L. Vese, Mumford and Shah model and its applications to image segmentation and image restoration, in Handbook of Mathematical Methods in Imaging, Springer-Verlag, Berlin, Heidelberg, 2011, pp. 1095-1157.

[8] S. Boyd, N. Parikh, E. Chu, B. Peleato, and J. Eckstein, Distributed optimization and statistical learning via the alternating direction method of multipliers, Found. Trends Mach. Learn., 3 (2011), pp. 1-122.

[9] X. Bresson, S. Esedō̄lu, P. Vandergheynst, J. Thiran, and S. Osher, Fast global minimization of the active contour/snake model, J. Math. Imaging Vision, 28 (2007), pp. 151-167. 
[10] E. S. Brown, T. F. Chan, And X. Bresson, A Convex Relaxation Method for a Class of Vector-Valued Minimization Problems with Applications to Mumford-Shah Segmentation, UCLA CAM Report (1043), UCLA, Los Angeles, CA, 2010.

[11] X. CAI, R. Chan, AND T. ZEng, A two-stage image segmentation method using a convex variant of the Mumford-Shah model and thresholding, SIAM J. Imaging Sci., 6 (2013), pp. 368-390.

[12] V. Caselles, A. Chambolle, and M. Novaga, The discontinuity set of solutions of the TV denoising problem and some extensions, Multiscale Model. Simul., 6 (2007), pp. 879-894.

[13] A. Chambolle, Image segmentation by variational methods: Mumford and Shah functional and the discrete approximations, SIAM J. Appl. Math., 55 (1995), pp. 827-863.

[14] A. Chambolle, Finite-differences discretization of the Mumford-Shah functional, M2AN Math. Model. Numer. Anal., 33 (1999), pp. 261-288.

[15] A. Chambolle, An algorithm for total variation minimization and applications, J. Math. Imaging Vision, 20 (2004), pp. 89-97.

[16] A. Chambolle and G. Dal Maso, Discrete approximation of the Mumford-Shah functional in dimension two, M2AN Math. Model. Numer. Anal., 33 (1999), pp. 651-672.

[17] A. Chambolle And T. Pock, A first-order primal-dual algorithm for convex problems with applications to imaging, J. Math. Imaging Vision, 40 (2011), pp. 120-145.

[18] T. F. Chan, S. Esedō̄uu, And M. Nikolova, Algorithms for finding global minimizers of image segmentation and denoising models, SIAM J. Appl. Math., 66 (2006), pp. 1632-1648.

[19] T. F. Chan, G. H. Golub, and P. Mulet, A nonlinear primal-dual method for total variation-based image restoration, SIAM J. Sci. Comput., 20 (1999), pp. 1964-1977.

[20] T. F. Chan And L. A. Vese, Active contours without edges, IEEE Trans. Image Process., 10 (2001), pp. 266-277.

[21] C. Chesnaud, P. RÉfréGier, And V. Boulet, Statistical region snake-based segmentation adapted to different physical noise models, IEEE Trans. Pattern Anal. Mach. Intell., 21 (1999), pp. 1145-1157.

[22] G. DAvid, Singular Sets of Minimizers for the Mumford-Shah Functional, Progr. Math. 233, Birkhäuser Verlag, Basel, 2005.

[23] B. Dong, A. Chien, And Z. Shen, Frame based segmentation for medical images, Commun. Math. Sci., 9 (2011), pp. 551-559.

[24] S. Durand, J. FAdili, And M. Nikolova, Multiplicative noise removal using L1 fidelity on frame coefficients, J. Math. Imaging Vision, 36 (2010), pp. 201-226.

[25] I. Ekeland And R. TÉmam, Convex Analysis and Variational Problems, Classics Appl. Math. 28, SIAM, Philadelphia, 1999.

[26] S. Esedō̄Lu And Y. Tsai, Threshold dynamics for the piecewise constant Mumford-Shah functional, J. Comput. Phys., 211 (2006), pp. 367-384.

[27] L. C. Evans, Partial Differential Equations, American Mathematical Society, Providence, RI, 1998.

[28] M. Feigin And N. Sochen, Segmentation and denoising via an adaptive threshold Mumford-Shah-like functional, in Proceedings of the 17th International Conference on Pattern Recognition, Vol. 2, IEEE Computer Society, Washington, DC, 2004, pp. 98-101.

[29] M. Figueiredo And J. Bioucas-Dias, Restoration of Poissonian images using alternating direction optimization, IEEE Trans. Image Process., 19 (2010), pp. 3133-3145.

[30] F. Galland, N. Bertaux, And P. RÉFrÉGier, Multi-component image segmentation in homogeneous regions based on description length minimization: Application to speckle, Poisson and Bernoulli noise, Pattern Recogn., 38 (2005), pp. 1926-1936.

[31] M. GobBino, Finite difference approximation of the Mumford-Shah functional, Comm. Pure Appl. Math., 51 (1998), pp. 197-228.

[32] T. Goldstein And S. OsheR, The split Bregman algorithm for L1-regularized problems, SIAM J. Imaging Sci., 2 (2009), pp. 323-343.

[33] L. Grady And C. Alvino, Reformulating and optimizing the Mumford-Shah functional on a graphA faster, lower energy solution, in Proceedings of the European Conference on Computer Vision (ECCV), Springer-Verlag, Berlin, Heidelberg, 2008, pp. 248-261.

[34] J. A. Hartigan and M. A. Wong, Algorithm AS 136: A K-means clustering algorithm, J. Roy. Statist. Soc. Ser. C. Appl. Stat., 28 (1979), pp. 100-108.

[35] M. Hintermüller and G. Stadler, An infeasible primal-dual algorithm for total bounded variationbased inf-convolution-type image restoration, SIAM J. Sci. Comput., 28 (2006), pp. 1-23. 
[36] T. Kanungo, D. Mount, N. Netanyahu, C. Piatko, R. Silverman, and A. Wu, An efficient Kmeans clustering algorithm: Analysis and implementation, IEEE Trans. Pattern Anal. Mach. Intell., 24 (2002), pp. 881-892.

[37] Y. KIM, Strictly Convex Realization in Two-Phase Image Segmentation, UCLA CAM Report (13-20), UCLA, Los Angeles, CA, 2013.

[38] G. Koepfler, C. Lopez, And J. M. Morel, A multiscale algorithm for image segmentation by variational method, SIAM J. Numer. Anal., 31 (1994), pp. 282-299.

[39] T. Le, R. Chartrand, And T. J. Asaki, A variational approach to reconstructing images corrupted by Poisson noise, J. Math. Imaging Vision, 27 (2007), pp. 257-263.

[40] J. Lellmann And C. SchnörR, Continuous multiclass labeling approaches and algorithms, SIAM J. Imaging Sci., 4 (2011), pp. 1049-1096.

[41] F. Li, M. K. NG, T. Y. Zeng, And C. Shen, A multiphase image segmentation method based on fuzzy region competition, SIAM J. Imaging Sci., 3 (2010), pp. 277-299.

[42] F. Li, C. Shen, AND C. LI, Multiphase soft segmentation with total variation and $H^{1}$ regularization, J. Math. Imaging Vision, 37 (2010), pp. 98-111.

[43] J. Lie, M. Lysaker, And X. TAI, A binary level set model and some applications to Mumford-Shah image segmentation, IEEE Trans. Image Process., 15 (2006), pp. 1171-1181.

[44] L. MA, M. K. NG, J. Yu, AND T. ZENG, Efficient box-constrained TV-type-l ${ }^{1}$ algorithms for restoring images with impulse noise, J. Comput. Math., 31 (2013), pp. 249-270.

[45] J. MacQueEn, Some methods for classification and analysis of multivariate observations, in Proceedings of the Fifth Berkeley Symposium on Mathematical Statistics and Probability, University of California Press, Berkeley, Los Angeles, 1967, pp. 281-297.

[46] M. Makitalo And A. FoI, Optimal inversion of the Anscombe transformation in low-count Poisson image denoising, IEEE Trans. Image Process., 20 (2011), pp. 99-109.

[47] D. Martin, C. Fowlkes, D. TaL, And J. Malik, A database of human segmented natural images and its application to evaluating segmentation algorithms and measuring ecological statistics, in Proceedings of the Eighth IEEE International Conference on Computer Vision, Vol. 2, 2001, pp. 416-423.

[48] P. Martin, P. RÉfrégier, F. Goudail, and F. Guérault, Influence of the noise model on level set active contour segmentation, IEEE Trans. Pattern Anal. Mach. Intell., 26 (2004), pp. 799-803.

[49] M. Morini And M. Negri, Mumford-Shah functional as $\Gamma$-limit of discrete Perona-Malik energies, Math. Models Methods Appl. Sci., 13 (2003), pp. 785-805.

[50] D. Mumford And J. Shah, Boundary detection by minimizing functionals, in Proceedings of the IEEE Conference on Computer Vision and Pattern Recognition, 1985, pp. 22-26.

[51] D. Mumford And J. Shah, Optimal approximations by piecewise smooth functions and associated variational problems, Comm. Pure Appl. Math., 42 (1989), pp. 577-685.

[52] T. Pock, A. Chambolle, D. Cremers, and H. Bischof, A convex relaxation approach for computing minimal partitions, in Proceedings of the IEEE Computer Society Conference on Computer Vision and Pattern Recognition (CVPR), 2009, pp. 810-817.

[53] T. Pock, D. Cremers, H. Bischof, and A. Chambolle, An algorithm for minimizing the MumfordShah functional, in Proceedings of the 2009 IEEE 12th International Conference on Computer Vision, 2009, pp. 1133-1140.

[54] O. Ruch AND P. RÉfrÉGIER, Minimal-complexity segmentation with a polygonal snake adapted to different optical noise models, Opt. Lett., 26 (2001), pp. 977-979.

[55] L. Rudin, P.-L. Lions, And S. Osher, Multiplicative denoising and deblurring: Theory and algorithms, in Geometric Level Set Methods in Imaging, Vision, and Graphics, Springer, New York, 2003, pp. 103119.

[56] L. I. Rudin, S. Osher, And E. FAtemi, Nonlinear total variation based noise removal algorithms, Phys. D, 60 (1992), pp. 259-268.

[57] B. Sandberg, S. KAng, And T. Chan, Unsupervised multiphase segmentation: A phase balancing model, IEEE Trans. Image Process., 19 (2010), pp. 119-130.

[58] A. Sawatzky, D. Tenbrinck, X. Jiang, and M. Burger, A variational framework for region-based segmentation incorporating physical noise models, J. Math. Imaging Vision, 47 (2013), pp. 179-209.

[59] B. Shafei And G. Steidl, Segmentation of images with separating layers by fuzzy c-means and convex optimization, J. Vis. Commun. Image Rep., 23 (2012), pp. 611-621. 
[60] J. SHi AND S. Osher, A nonlinear inverse scale space method for a convex multiplicative noise model, SIAM J. Imaging Sci., 1 (2008), pp. 294-321.

[61] G. Steidl and T. Teuber, Removing multiplicative noise by Douglas-Rachford splitting methods, J. Math. Imaging Vision, 36 (2010), pp. 168-184.

[62] A. Tsai, A. Yezzi, ANd A. Willsky, Curve evolution implementation of the Mumford-Shah functional for image segmentation, denoising, interpolation, and magnification, IEEE Trans. Image Process., 10 (2001), pp. 1169-1186.

[63] L. Vese And T. Chan, A multiphase level set framework for image segmentation using the Mumford and Shah model, Int. J. Comput. Vis., 50 (2002), pp. 271-293.

[64] Y.-W. WEN AND R. H. Chan, Parameter selection for total-variation-based image restoration using discrepancy principle, IEEE Trans. Image Process., 21 (2012), pp. 1770-1781.

[65] J. YuAn, E. BAE, AND X.-C. TAI, A study on continuous max-flow and min-cut approaches, in Proceedings of the Twenty-Third IEEE Conference on Computer Vision and Pattern Recognition (CVPR), San Francisco, CA, 2010, pp. 2217-2224.

[66] J. Yuan, E. Bae, X.-C. TAi, And Y. Boykov, A continuous max-flow approach to Potts model, in Computer Vision-ECCV 2010, Springer, Berlin, Heidelberg, 2010, pp. 379-392.

[67] J. Yuan, C. Schnörr, And G. Steide, Simultaneous higher-order optical flow estimation and decomposition, SIAM J. Sci. Comput., 29 (2007), pp. 2283-2304.

[68] M. Zhu and T. Chan, An Efficient Primal-Dual Hybrid Gradient Algorithm for Total Variation Image Restoration, UCLA CAM Report (08-34), UCLA, Los Angeles, CA, 2008. 